\title{
Optical spectroscopic characterizations of laser irradiated olivine grains
}

\author{
Yazhou Yang (杨亚洲 $)^{1}$, Hao Zhang (张昊 $)^{1,2}$, Ziwei Wang (王紫薇 $)^{1}$, Ye Yuan (袁野) ${ }^{1}$, Shaolin Li (李少林) ${ }^{3}$, \\ Weibiao Hsu (徐伟彪) ${ }^{3}$, and Chujian Liu (刘初见 $)^{4}$ \\ 1 Planetary Science Institute, School of Earth Sciences, China University of Geosciences, Wuhan 430074, PR China \\ e-mail: um_zhanghao@yahoo.com; yangyazhou1@gmail.com \\ 2 Key Laboratory of Spectral Imaging Technology, Xi' an Institute of Optics and Precision Mechanics, Chinese Academy of Sciences, \\ Xi' an 710119, PR China \\ 3 Purple Mountain Observatory, Chinese Academy of Sciences, Nanjing, PR China \\ ${ }^{4}$ State Key Laboratory of Geological Process and Mineral Resources, China University of Geosciences, Wuhan, PR China
}

Received 16 July 2016 / Accepted 11 October 2016

\begin{abstract}
Context. Visible and near-infrared spectra of asteroids are known to be susceptible to nanophase irons produced by space weathering processes, thus making mineral identifications difficult. Mid-infrared spectroscopy may retain more mineral features owing to its lattice vibrational nature.

Aims. We investigate the structure and reflectance spectral feature changes of olivine grains before and after simulated space weathering.

Methods. We irradiate olivine grains by using pulsed laser to simulate varying degrees of micrometeorite bombardments. Reflectance measurements from 0.5 to $25 \mu \mathrm{m}$ and radiative transfer calculations were carried out in order to compare them with each other.

Results. Both the experimental simulations and modeling results indicate that the mid-infrared spectral features of olivine grains can survive the intense irradiations. Although the Christansen Feature is slightly shifted to longer wavelength, major vibrational bands remain essentially unchanged, because the lattice structure is quite immune to even the strongest irradiations, as revealed by both the $\mathrm{X}$-ray diffraction and Raman scattering measurements.

Conclusions. Mid-infrared spectroscopy is much more immune to productions of nanophase irons and amorphous materials and thus may be used more reliably in remote detections of minerals on asteroid surfaces.
\end{abstract}

Key words. methods: laboratory: solid state - techniques: spectroscopic - radiative transfer - planets and satellites: surfaces infrared: general - planets and satellites: composition

\section{Introduction}

Visible and near-infrared (VNIR) reflectance spectroscopy has been widely used in characterizing the surface mineralogical compositions of airless bodies as most silicate minerals have diagnostic absorption features in the VNIR region (e.g., Chapman 1996, 2004; Gaffey 2010). However, space weathering processes including micrometeorite impacts and ion implantations may darken and redden the VNIR spectra, reduce the spectral contrast, and thus make the spectral interpretations difficult (e.g., Hapke 2001; Chapman 2004; Nesvornỳ et al. 2005). Both laboratory simulations and returned-sample analysis have demonstrated that nanophase iron particles $\left(\mathrm{npFe}^{0}\right)$, produced by the redeposition of vapor generated by the sputtering of solar wind particles and micrometeorite impacts (e.g., Hapke et al. 1975; Keller \& McKay 1993; Hapke 2001), are mainly responsible for the optical effects of space weathering (e.g., Keller \& McKay 1993; Pieters et al. 2000; Taylor et al. 2001; Sasaki et al. 2001, 2002, 2003; Brunetto et al. 2006; Loeffler et al. 2008; Britt et al. 2014).

Currently, laboratory simulations of space weathering can be categorized into three main types: pulsed laser is used to irradiate analog materials to simulate effects of micrometeorite impacts (e.g., Yamada et al. 1999; Sasaki et al. 2001; Brunetto et al. 2006); proton and ion implantations are used to simulate solar-wind irradiations (e.g., Hapke 1973; Yamada et al. 1999; Brucato et al. 2004; Marchi et al. 2005; Fulvio et al. 2012; Miller et al. 2014; Kuhlman et al. 2015); and thermal treatment is also used to simulate the production of $\mathrm{npFe}^{0}$ (e.g., Tang et al. 2012). Recently, Kohout et al. (2014) developed a double heating method to produce $n \mathrm{pFe}^{0}$ in a controlled way. Among these methods, pulsed laser irradiation is very effective in producing the spectral effects of space weathering as the short pulse can deliver energy in a very short timescale which is believed to be comparable to the impact duration of micrometeorites (e.g., Yamada et al. 1999).

The effects of $n p \mathrm{Fe}^{0}$ on VNIR spectra have been thoroughly studied since the confirmation of its existence on returned lunar regolith particles. Because the VNIR features below $2.5 \mu \mathrm{m}$ in olivine and pyroxene spectra are mainly caused by electronic transitions of ferrous ions in crystal field (Burns 1993), the VNIR spectra may be greatly affected by the production of $n p F e^{0}$. The VNIR spectral effects of the $n p \mathrm{Fe}^{0}$ with varying contents, distributions, and particle sizes have been studied by using the Hapke radiative transfer model with some success (e.g., Hapke 2001; Lawrence \& Lucey 2007; Denevi et al. 2008). In 
contrast, space weathering effects on reflectance bands at longer wavelengths caused by thermal lattice vibrations are less well studied, but may contain rich information on space weathered minerals (Lucey et al. 2016). Early studies before modern space weathering simulations were carried out show that space weathering causes little mid-infrared (MIR) spectral changes (Salisbury et al. 1997), though this view was challenged recently (Lucey et al. 2016).

In the far-infrared region where the radiation wavelength is longer than $25 \mu \mathrm{m}$ (wavenumber below $400 \mathrm{~cm}^{-1}$ ), the reflectance peaks are caused by external lattice modes such as the relative motions between the ferrous ions and the $\mathrm{SiO}_{4}$ group. Reflectance bands in the MIR from $10 \mu \mathrm{m}$ to $25 \mu \mathrm{m}$ (1000 to $400 \mathrm{~cm}^{-1}$ ) are the so-called fundamental vibrational modes (Poulet \& Mathieu 1976) that can be divided into internal stretching, bending and librating of the $\mathrm{SiO}_{4}$ group. In the short wavelength infrared (SWIR) region roughly between $2.5 \mu \mathrm{m}$ and $10 \mu \mathrm{m}$, reflectance peaks are mainly overtones and combinational bands of the fundamental lattice modes. As a result, spectral features within these regions could be less susceptible to the production of $\mathrm{nFFe}^{0}$ and hence could be potentially useful in identifying the mineralogical compositions of the surfaces of airless bodies.

In the MIR region, some prominent spectral features such as the Christiansen feature (CF), the Restrahlen bands and the transparency feature (TF) may be used as diagnostic features of mineral compositions (e.g., Mustard \& Hays 1997; Pieters 1998). The CF of silicates, manifesting itself as the reflectance minimum between 7.5 and $9 \mu \mathrm{m}$, occurs near the Christiansen wavelength where the real part of refractive index approaches unity (e.g., Mustard \& Hays 1997; Hapke 2012). The restrahlen bands in silicates include one stronger band between 8.5 and $12 \mu \mathrm{m}$ and one less strong band between 16.5 and $25 \mu \mathrm{m}$, caused by $\mathrm{Si}-\mathrm{O}$ stretching and $\mathrm{Si}-\mathrm{O}-\mathrm{Si}$ bending vibrations, respectively (e.g., Noel et al. 2006). The TF is a reflectance maximum located in a volume-scattering region between the two restrahlen bands described above. The CF and TF are correlated with each other and are both dependent on compositions (e.g., Hapke 2012).

Although observational data above $2 \mu \mathrm{m}$ are mostly thermal emission spectra, reflectance spectroscopy is a powerful and convenient tool in the laboratory to study mineral optical properties. Furthermore, the emission and reflectance spectra contain the same spectral features and are complementary through the Kirchhoff's law (Hapke 2012), and thus MIR reflectance spectroscopy is widely used to understand minerals' micro-structures (e.g., Pieters 1998, 2002). Another benefit of using the MIR spectroscopy is that it may potentially provide complementary information to the VNIR spectra. For example, Beichman et al. (2005) identified crystalline olivine based on the diagnostic features between 8 and $35 \mu \mathrm{m}$. In addition, it has been found that some VNIR spectroscopically similar asteroids display distinctive spectral features in the MIR (Vernazza et al. 2010).

To understand the MIR characteristics of planetary ana$\log$ materials before and after space weathering, we carried out pulsed laser irradiation simulations and reflectance measurements of olivine grains. In order to understand the simulation results semi-quantitatively, we also performed reflectance model predictions by using both a semi-empirical reflectance model (Hapke 2012) and a strict numerical radiative transfer model (Stamnes et al. 2000). We first present the simulation experimental procedures and the modeling methods, followed by results and discussion sections.
Table 1. Chemical composition of the original and the VDM processed olivine samples.

\begin{tabular}{ccc}
\hline \hline $\begin{array}{c}\text { Major } \\
\text { elements }\end{array}$ & $\begin{array}{c}\text { Original olivine } \\
(\text { wt. \%) }\end{array}$ & $\begin{array}{c}\text { Olivine processed } \\
\text { with VDM (wt. \%) }\end{array}$ \\
\hline $\mathrm{SiO}_{2}$ & 40.660 & 40.980 \\
$\mathrm{Al}_{2} \mathrm{O}_{3}$ & 0.340 & 0.230 \\
$\mathrm{TFe}_{2} \mathrm{O}_{3}$ & 9.760 & 9.780 \\
$\mathrm{MgO}$ & 48.000 & 48.590 \\
$\mathrm{CaO}$ & 0.056 & 0.042 \\
$\mathrm{Na}_{2} \mathrm{O}$ & 0.008 & 0.010 \\
$\mathrm{~K}_{2} \mathrm{O}$ & 0.004 & 0.003 \\
$\mathrm{TiO}_{2}$ & 0.011 & 0.005 \\
$\mathrm{P}_{2} \mathrm{O}_{3}$ & 0.005 & 0.012 \\
$\mathrm{MnO}^{\mathrm{H} O}$ & 0.110 & 0.110 \\
$\mathrm{H}_{2} \mathrm{O}$ & 0.040 & 0.040 \\
\hline $\mathrm{Total}$ & 98.994 & 99.802 \\
\hline ** Fo\# & 95 & 95 \\
\hline
\end{tabular}

Notes. ${ }^{(*)}$ Total iron; ${ }^{(* *)}$ Mg-number.

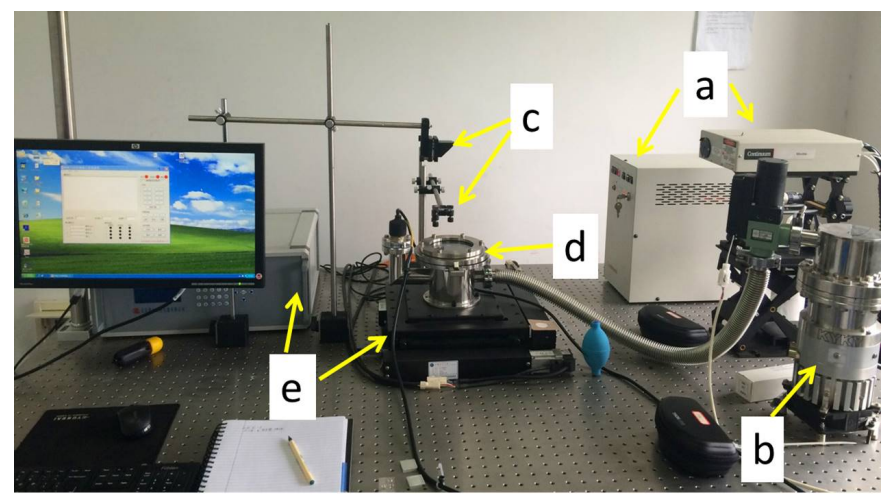

Fig. 1. Experimental setup of pulsed laser irradiations. a) Nd:YAG pulsed laser; b) molecular pump; c) reflector and optical lens; d) vacuum chamber; e): motorized $X-Y$ translation stage and the controller.

\section{Materials and methods}

\subsection{Experimental procedure}

Samples. Olivine, a common mineral found in many meteorites and S-type asteroids (e.g., Chapman 1996, 2004), was used as the analog material in this work. Natural pure olivine granules collected from Hebei Province, China, were first grinded into a size distribution of $0 \sim 75 \mu \mathrm{m}$ with a Vibratory Disc Mill (VDM). The olivine powders were then sieved and those smaller than $45 \mu \mathrm{m}$ were separated as the target sample. The chemical compositions of these olivine powders were analyzed with wet chemistry method. To ensure that no contaminations were introduced during the grinding process, compositional measurements were made on both the original samples and the powders processed with the VDM. The contents of major elements of these two samples, as summarized in Table 1, indicate that the grinding process did not introduce any contaminations of iron, and the $\mathrm{Mg}$ number of the olivine sample is 95 (Fo95).

Pulsed laser irradiations. Figure 1 shows the irradiation system used in our simulation experiments (Yamada et al. 1999; Sasaki et al. 2001). A 1064 nm Continuum Minilite II nanosecond pulsed laser with incident energy per pulse from 1 to $50 \mathrm{~mJ}$ 
was used to simulate the micrometeorite bombardment process. After being focused by a thin lens, the laser beam diameter is reduced from $3 \mathrm{~mm}$ to $0.5 \mathrm{~mm}$ to increase the energy density. The pulse duration is $6 \sim 7$ nanoseconds which is believed to be comparable with the timescale of real micrometeorite impacts (Yamada et al. 1999). Samples were placed in aluminum holders in the vacuum chamber under a pressure of $10^{-3} \mathrm{~Pa}$. To remove moisture in the samples as much as possible, the powdery sample was baked for more than $5 \mathrm{~h}$ under $120^{\circ} \mathrm{C}$ in a vacuum oven before each irradiation was carried out. Each time $0.2 \mathrm{~g}$ of olivine powders were uniformly placed on a $25 \mathrm{~mm} \times 25 \mathrm{~mm}$ square area. A thin glass slide was used to cover the holder to prevent the sputtering of the powders. The chamber was installed on a motorized $X-Y$ translation stage which is controlled by a computer. By programming the stage movement the powdery samples can be uniformly irradiated. In this work, samples were irradiated with a frequency of $15 \mathrm{~Hz}$ and a scanning velocity of $1 \mathrm{~mm}$ per second. By changing the pulse energy and number of irradiations, products with different weathering degrees can be obtained. Two pulse energy levels, 25 and $50 \mathrm{~mJ} /$ pulse, were used, yielding a total energy density of 750 and $1500 \mathrm{~mJ} / \mathrm{mm}^{2}$ for one irradiation experiment, respectively. We chose these two energy levels for the following reason. For an airless body located at $1 \mathrm{AU}$, the impact energy imparted by a $1 \mu \mathrm{m}$ dust particle with mass of $10^{-12} \mathrm{~g}$ moving at $20 \mathrm{~km} \mathrm{~s}^{-1}$ towards its surface is about $2 \times 10^{-4} \mathrm{~mJ}$ (Yamada et al. 1999), giving an energy density of $\sim 250 \mathrm{~mJ} / \mathrm{mm}^{2}$ which is equivalent to that of one single $50 \mathrm{~mJ}$ pulse in our experiments. Therefore, with a frequency of $15 \mathrm{~Hz}$, even the $25 \mathrm{~mJ}$ level should be strong enough to simulate both lunar and asteroidal space weathering styles caused by micrometeorite bombardment (e.g., Yamada et al. 1999; Sasaki et al. 2001).

Reflectance measurements. After laser irradiations, the powdery sample was removed from the vacuum chamber immediately to have its VNIR and MIR reflectance spectra measured by a Bruker Vertex 70 Fourier transform infrared spectrometer equipped with Easy Diffuse accessories from Pike Technologies. The VNIR spectra were calibrated with a Labsphere Spectralon plaque with a $99 \%$ nominal reflectance in the visible. For MIR measurements, a gold mirror was used as the reference as the Spectralon has numerous absorption peaks in this spectral region. Both the VNIR and MIR spectra were measured under a biconical configuration with both the incident and viewing zenith angles spanning within the range between $40^{\circ}$ to $65^{\circ}$. To reduce any possible spectral variations caused by surface inhomogeneities and packing structures, the sample holder was rotated 90 degrees after each measurement and altogether four measurements were made. When the maximum difference among the four measurements was within 5\%, the measurements were deemed as reliable and the average of the four was used as the final result; otherwise a new surface was made to be measured.

Structural and compositional analysis. To verify the microscopic changes of the olivine particles, we performed structural analysis on some irradiated samples by using a Titan G2 60-300 Transmission Electron Microscope (TEM) equipped with an Energy Dispersive Spectrometer (EDS). We adopted the ultrasonic dispersion method by adding the powdery sample into anhydrous ethanol to prepare samples for TEM analysis.

In addition to the TEM observations, we also performed powder X-ray Diffraction (XRD) and Raman spectroscopic measurements for all the olivine samples to check any possible crystal structural changes. The XRD measurements were done on a Bruker AXS D8-Focus X-ray Diffractometer. Raman scattering was carried out by using a Thermo Scientific DXR microRaman system with a spectral resolution of $1.5-2 \mathrm{~cm}^{-1}$. The excitation laser wavelength was $532 \mathrm{~nm}$ and the incident power was varied from 1 to $9 \mathrm{~mW}$ to exclude any possible laser power dependent spectral changes.

\subsection{Reflectance spectra modeling}

Based on the results of our simulation experiments and previous studies, we performed reflectance model predictions to understand the spectral feature changes. $\mathrm{npFe}^{0}$ bearing rims on returned lunar (Keller \& McKay 1997) and asteroid Itokawa (Noguchi et al. 2011) regolith particles are now known to be responsible for the spectral changes of darkening and reddening. Laboratory simulations with analog materials have successfully reproduced $n \mathrm{pFe}^{0}$ production and the incurred reddening and darkening effects on VNIR spectra (e.g., Yamada et al. 1999; Sasaki et al. 2001; Brunetto et al. 2006). In addition to $\mathrm{npFe}^{0}$, a glassy coating or amorphous layer is also produced during space weathering processes (Keller \& McKay 1997; Noguchi et al. 2011; Berger \& Keller 2015). To understand the spectral effects of these two factors, we have performed reflectance model predictions using the Hapke photometric model (Hapke 2012) and a strict numerical algorithm, the discrete ordinate radiative transfer (DISORT) model (Stamnes et al. 2000).

The Hapke model used here (Hapke 1981) is a simplified version without the opposition effect as the current spectroscopic measurements were made at large phase angles. In this work, the bidirectional reflectance $r$ is expressed as

$r(i, e, g)=\frac{\omega}{4 \pi} \frac{\mu_{0}}{\mu_{0}+\mu}\left[p(g)+H\left(\gamma, \mu_{0}\right) H(\gamma, \mu)-1\right]$,

where $i, e$, and $g$ are the incidence, emission, and phase angles, respectively. $\mu_{0}=\cos (i), \mu=\cos (e), \omega$ is the single scattering albedo, $p(g)$ is the particle phase function, and $H(\gamma, \mu)$ is the Ambartsumian-Chandrasekha $H$ function. An analytic approximation of $H(\gamma, x)$ is given by

$H(\gamma, x) \approx \frac{1+2 x}{1+2 \gamma x}$

where $\gamma$ is the albedo factor defined as

$\gamma=\sqrt{1-\omega}$.

If the single scattering quantities $\omega$ and $p(g)$ are known, other terms related to $\omega$ can be calculated and then we can obtain the reflectance quantities. In this work, we present both the model data and measurements in terms of reflectance defined as the ratio of the sample's bidirectional reflectance to that of a perfect Lambertian surface,

Reflectance $=\frac{r(i, e, g) \pi}{\mu_{0}}$.

Since the performance of the Hapke model has been in constant debate (e.g., Zhang \& Voss 2011; Shkuratov et al. 2012; Hapke 2013), we also employed the DISORT program which numerically solves the radiative transfer equation and can achieve very high numerical precisions with enough "streams" (Stamnes et al. 2000). Although it was mainly intended for scattering media with negligible close packing effects such as atmospheric 


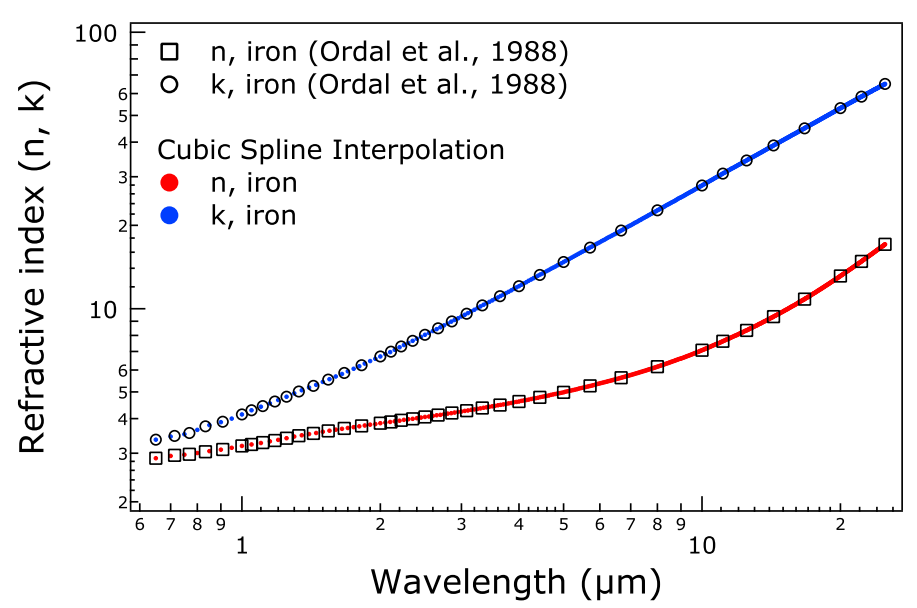

Fig. 2. Original and interpolated refractive indices $n$ (real part) and $k$ (imaginary part) of iron (Ordal et al. 1988).

aerosols, it was found to be able to predict reflectance measurements on closely packed transparent spherical particles very well except in the backscattering direction (Zhang \& Voss 2011). We incorporate both the semi-empirical model and the strict numerical model to better understand reflectance predictions on laser irradiated grains.

The single scattering quantities $\omega$ and $p(g)$ needed by both models were computed by Mie algorithm (Mishchenko et al. 1999) for uniform particles, and the BART code (Quirantes \& Bernard 2004) for grains coated with amorphous layers or $\mathrm{nFe}^{0}$ rim, respectively. The input parameters of both codes include complex refractive indices and particle size distribution parameters. The representative particle size used in this study was assumed to be $30 \mu \mathrm{m}$ in diameter. The refractive index data of olivine, amorphous materials (silicate glass) and metal iron needed in the calculations are from Fabian et al. (2001), Dorschner et al. (1995), Jäger et al. (1994), and Ordal et al. (1988), respectively. To achieve a higher spectral resolution, the original sparse discrete and unevenly spaced data points were interpolated to a spectral resolution of $0.05 \mu \mathrm{m}$ between 0.65 and $25 \mu \mathrm{m}$ using the cubic spline interpolation algorithm. Figure 2 shows the original and interpolated refractive indices of iron. Figure 3 a shows the interpolated $n$ and $k$ data of four different silicate glasses, two pyroxene (Glass 1: $\mathrm{Mg}_{0.95} \mathrm{Fe}_{0.05} \mathrm{SiO}_{3}$, Glass 2: $\mathrm{Mg}_{0.4} \mathrm{Fe}_{0.6} \mathrm{SiO}_{3}$ ) and two olivine (Glass 3: $\mathrm{Mg}_{2} \mathrm{SiO}_{4}$, Glass 4: $\mathrm{Mg}_{0.8} \mathrm{Fe}_{1.2} \mathrm{SiO}_{4}$ ) types. We considered two pyroxene glass coating types in addition to two olivine types because during melting and evaporations the coating compositions might change. Since olivine belongs to orthorhombic symmetry, its refractive indices in the MIR vary with different orientations. Figures $3 \mathrm{~b}, \mathrm{c}$ show the $n$ and $k$ data along three crystallographic axes $x, y$ and $z$ of Fo95 olivine (Fabian et al. 2001). We made computations with these optical constants of $x, y$ and $z$ axes, and use their average to approximate the reflectance of randomly orientated crystals (e.g., Brucato et al. 2004).

For particles with uniformly distributed $n \mathrm{pFe}^{0}$, we calculated the refractive index based on the Maxwell-Garnet effective medium theory (e.g., Bohren \& Huffman 2008; Hapke 2001) as

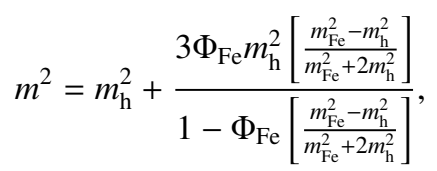

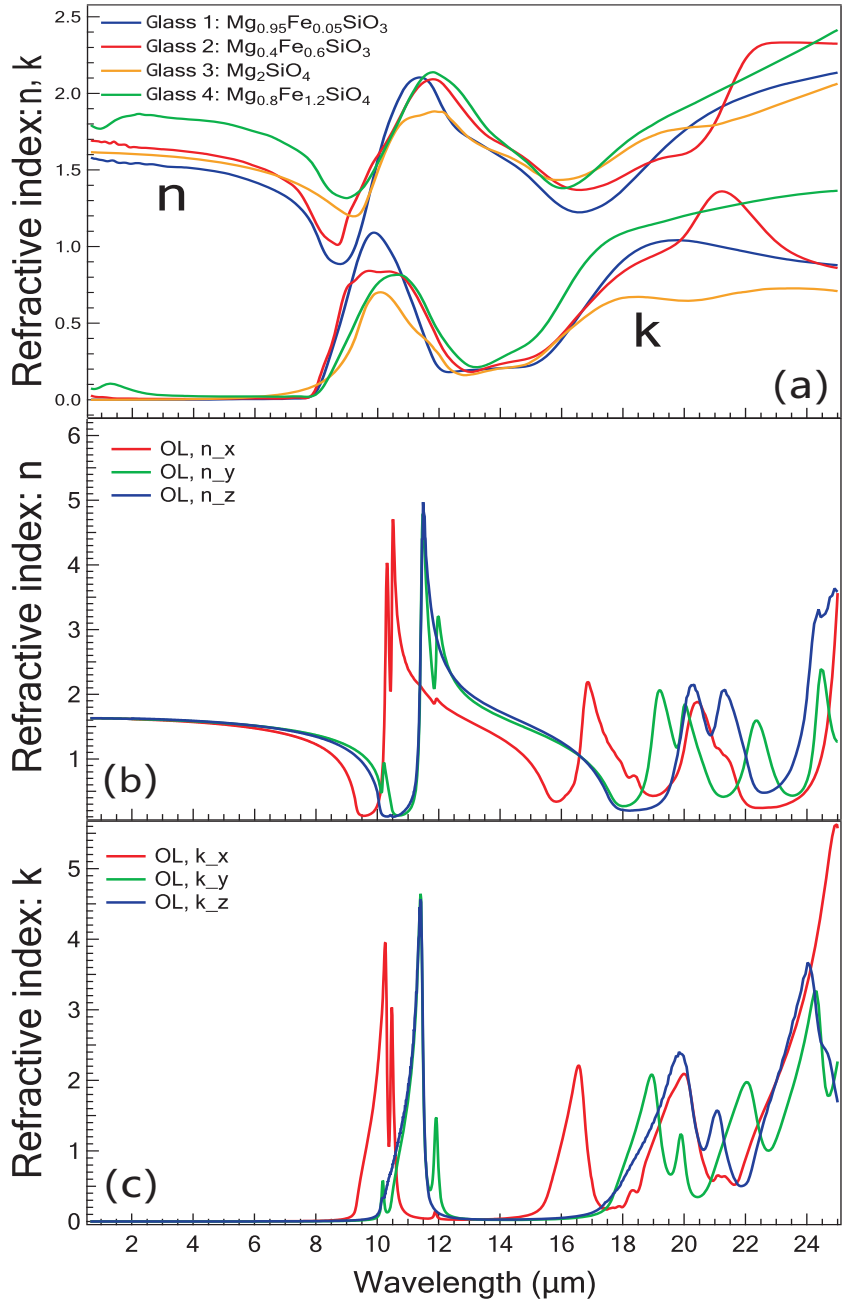

Fig. 3. Interpolated refractive indices $n$ and $k$ of olivine and four different silicate glasses. a) $n, k$ data of Glass 1 and Glass 2 from Jäger et al. (1994); Glass 3 from Fabian et al. (2000); and Glass 4 from Dorschner et al. (1995); b) $n$ data of olivine from Fabian et al. (2001); c) $k$ data of olivine from Fabian et al. (2001). $x, y$ and $z$ indicate three different crystallographic axes.

where $m$ is the effective complex refractive index, $m_{\mathrm{h}}=n_{\mathrm{h}}+\mathrm{i} k_{\mathrm{h}}$ and $m_{\mathrm{Fe}}=n_{\mathrm{Fe}}+\mathrm{i} k_{\mathrm{Fe}}$ are the refractive indices of host material and iron, respectively, $\Phi_{\mathrm{Fe}}$ is the volumetric fraction of the $n p \mathrm{pe}^{0}$.

\section{Results}

\subsection{Experimental results}

Figure 4 shows the original olivine sample and three typical samples irradiated with different pulse energy levels $(25$ and $50 \mathrm{~mJ})$ and duration combinations ( 2 and 5 times). When compared to the original fresh white sample (Fig. 4a), one easily sees the albedo reduction of samples irradiated under $25 \mathrm{~mJ} /$ pulse for 2 times (Fig. 4b). With the increase in energy level and number of irradiations, the darkening effect becomes stronger. As an extreme, the sample irradiated with $50 \mathrm{~mJ} /$ pulse for 5 times becomes charcoal black (Fig. 4d).

Reflectance spectra. The VNIR reflectance spectra of seven olivine samples irradiated with varying degrees and the unirradiated one are shown in Fig. 5, while the same spectra normalized at $750 \mathrm{~nm}$ are shown in Fig. 6. After irradiations the 
(a)

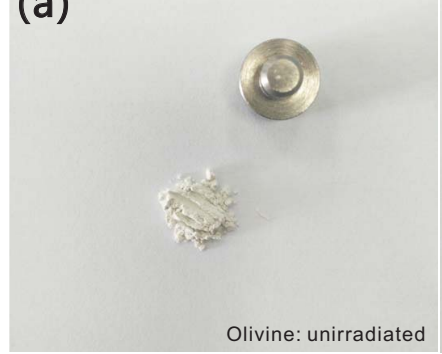

(c)

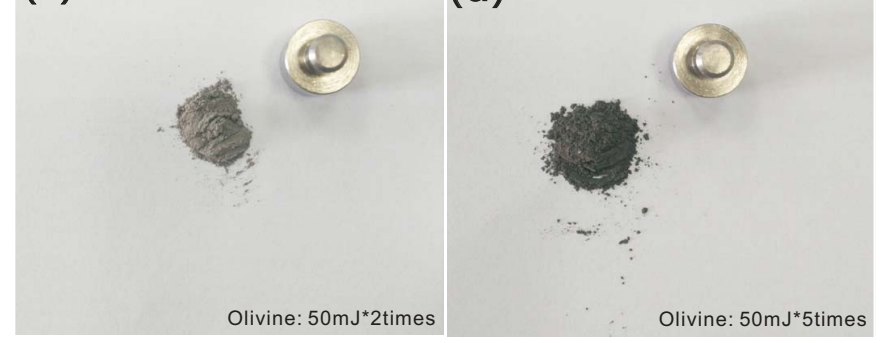

Fig. 4. Typical olivine powders before and after irradiations with different pulse energy levels and number of irradiations. a) Original olivine sample; b) after irradiation with $25 \mathrm{~mJ} /$ pulse for 2 times; c) after irradiation with $50 \mathrm{~mJ} /$ pulse for 2 times; d) after irradiation with $50 \mathrm{~mJ} /$ pulse for 5 times. The sample holder used in reflectance measurement is also shown as a reference.

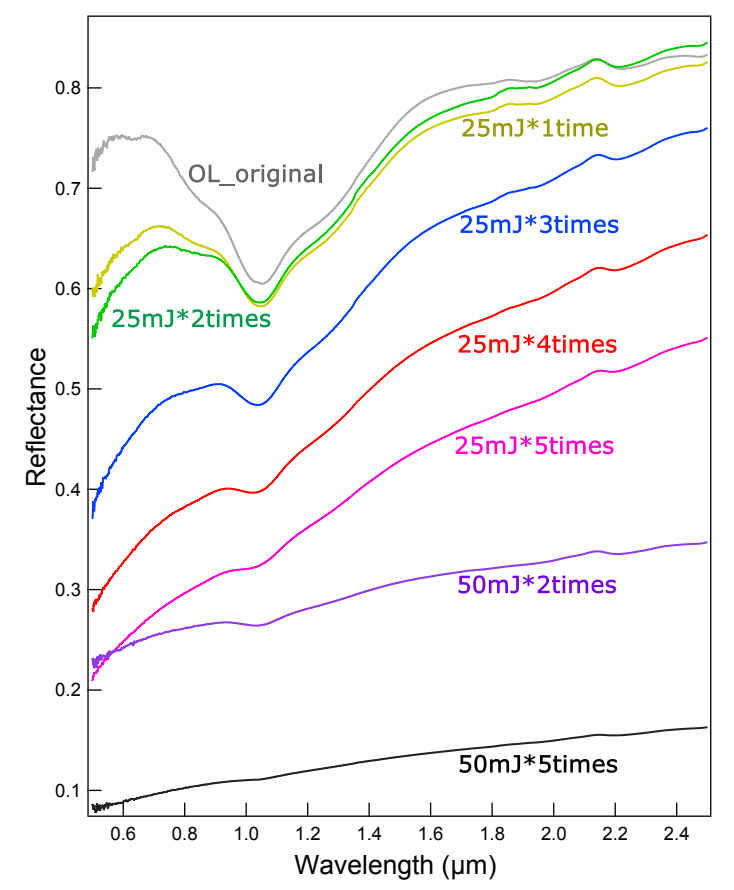

Fig. 5. VNIR reflectance spectra of olivine samples before and after irradiations. Eight spectra: non-irradiated, irradiated under $25 \mathrm{~mJ} /$ pulse for 1 to 5 times, irradiated under $50 \mathrm{~mJ} /$ pulse for 2 and 5 times. All spectra are measured relative to a Spectralon standard with $99 \%$ nominal reflectance.

VNIR spectra of olivine show the well-known darkening and reddening changes (e.g., Yamada et al. 1999; Sasaki et al. 2001). We characterize the VNIR spectral changes by using three types of parameters: the reflectance values at $600 \mathrm{~nm}$ and $1700 \mathrm{~nm}$ are used to describe the darkening effect in the visible and near-infrared (NIR) regions; the ratio of reflectance values at $1700 \mathrm{~nm}$ and $750 \mathrm{~nm}$ is used to characterize the reddening effect; the depth of the $1 \mu \mathrm{m}$ absorption band is used to evaluate

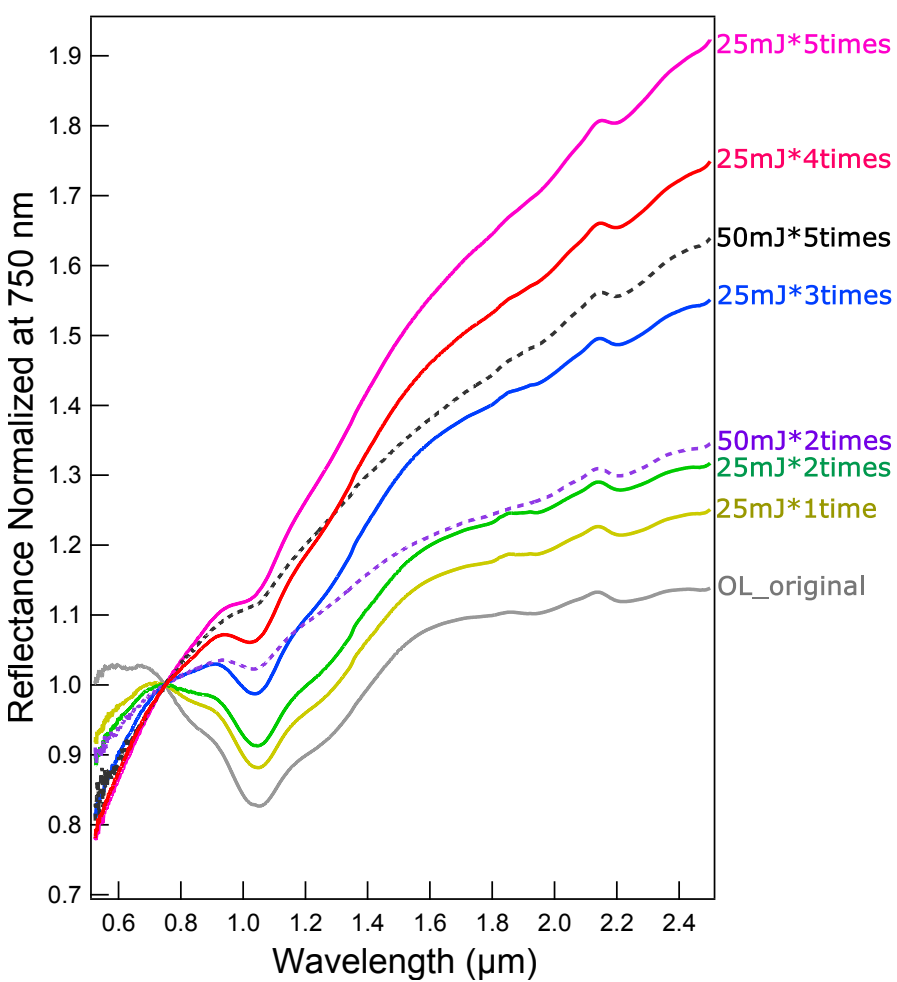

Fig. 6. VNIR reflectance spectra of olivine samples normalized at $750 \mathrm{~nm}$.
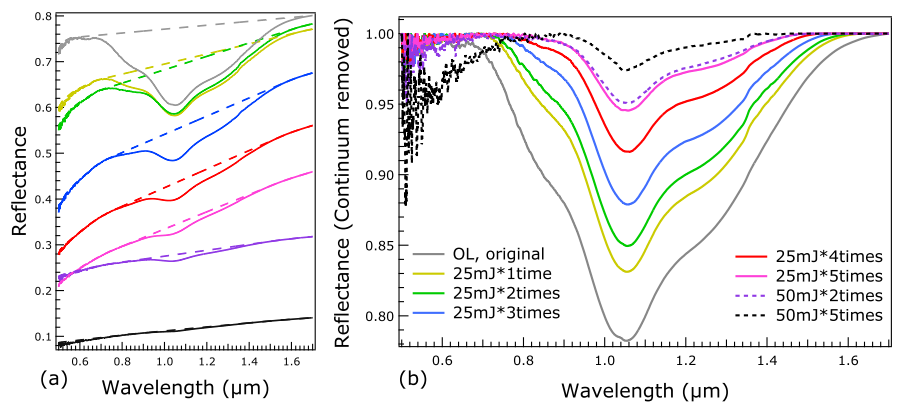

Fig. 7. Reflectance spectra before and after removing the continuum. a) The original spectra (solid) and the continua used as the baselines (dashed) obtained by fitting the peaks between 0.5 and $1.7 \mu \mathrm{m}$; b) the continuum-removed spectra.

the decrease in spectral contrast. The band depth is estimated from the continuum-removed spectra (Fig. 7) by subtracting the minimum reflectance value within the absorption band from unity (Clark \& Roush 1984). We limit the VNIR feature discussions in the spectral region $500 \sim 1700 \mathrm{~nm}$ as the spurious peak at $2.14 \mu \mathrm{m}$ caused by Spectralon absorption (Zhang et al. 2014) would affect the shape of the baseline. All VNIR spectral changes are summarized in Table 2.

Compared with the original olivine spectrum, all irradiated sample spectra show more reductions of reflectance in the visible than in the NIR. The normalized spectra shown in Fig. 6 are redder with the increase in number of irradiations. The depth of the $1 \mu \mathrm{m}$ absorption band of the original sample is 0.218 (Table 2) and it dropped to 0.054 and 0.049 after being irradiated under $25 \mathrm{~mJ} /$ pulse for 5 times and $50 \mathrm{~mJ} /$ pulse for 2 times, respectively. After being irradiated with $50 \mathrm{~mJ} /$ pulse for 5 times, the overall reflectance value decreased by more than $70 \%$, and the $1 \mu \mathrm{m}$ absorption feature almost disappeared completely (over irradiated). Irradiation with $50 \mathrm{~mJ} /$ pulse for 2 times caused more 

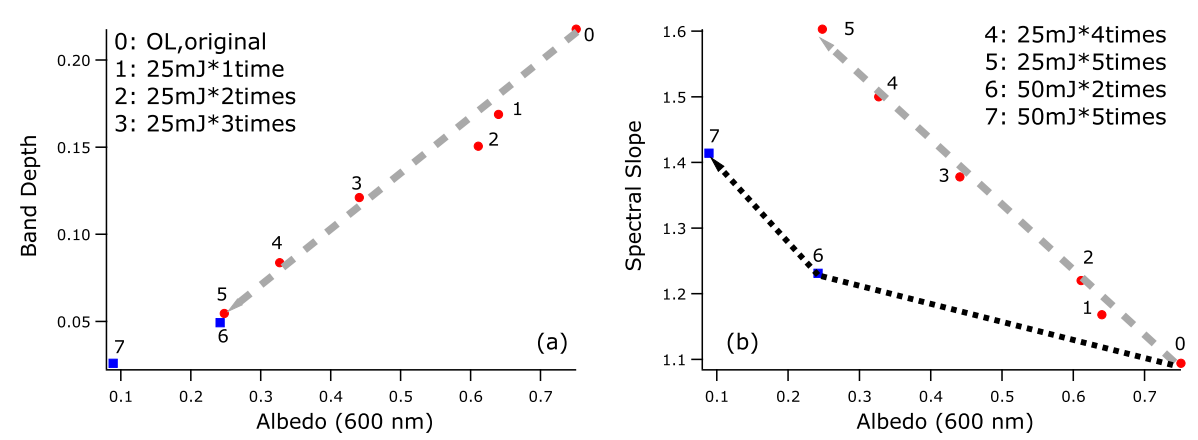

Fig. 8. Variations of the $1 \mu \mathrm{m}$ band depth and the VNIR spectral slope of olivines with the increased number of irradiations. a) The $1 \mu \mathrm{m}$ band depth vs. albedo at $600 \mathrm{~nm}$; b) the spectral slopes vs. albedo. The arrow at the end indicates the increase in irradiation levels.

Table 2. Parameters characterizing the VNIR spectral features of olivines before and after irradiations.

\begin{tabular}{|c|c|c|c|c|}
\hline \multirow{2}{*}{$\begin{array}{l}\text { Olivine } \\
\text { samples }\end{array}$} & \multicolumn{2}{|c|}{ Albedo } & \multirow{2}{*}{$\begin{array}{l}\text { Band } \\
\text { depth }\end{array}$} & \multirow{2}{*}{$\begin{array}{c}\text { Reddening } \\
R(1700) / R(750)\end{array}$} \\
\hline & $600 \mathrm{~nm}$ & $1700 \mathrm{~nm}$ & & \\
\hline Original & 0.751 & 0.801 & 0.218 & 1.094 \\
\hline $25 \mathrm{~mJ} \times 1$ time & 0.640 & 0.771 & 0.169 & 1.168 \\
\hline $25 \mathrm{~mJ} \times 2$ times & 0.611 & 0.783 & 0.150 & 1.220 \\
\hline $25 \mathrm{~mJ} \times 3$ times & 0.441 & 0.675 & 0.121 & 1.378 \\
\hline $25 \mathrm{~mJ} \times 4$ times & 0.327 & 0.561 & 0.084 & 1.500 \\
\hline $25 \mathrm{~mJ} \times 5$ times & 0.248 & 0.460 & 0.054 & 1.603 \\
\hline $50 \mathrm{~mJ} \times 2$ times & 0.242 & 0.318 & 0.049 & 1.231 \\
\hline $50 \mathrm{~mJ} \times 5$ times & 0.089 & 0.141 & 0.026 & 1.414 \\
\hline
\end{tabular}

prominent darkening than that with $25 \mathrm{~mJ} /$ pulse for 5 times did, especially in the NIR region.

Figure 8 shows how the band depth and spectral slope of the VNIR spectra change with the increase in number of irradiations. The spectral slope, albedo and band depth almost reduced simultaneously under irradiation with $25 \mathrm{~mJ} /$ pulse, corresponding to a typical lunar style weathering (Gaffey 2010). In contrast, irradiation with a higher energy level $(50 \mathrm{~mJ} /$ pulse) caused a quicker decrease in albedo and band depth and the decreases are not proportional to the number of irradiations.

It is noted that irradiations with $25 \mathrm{~mJ}$ for 2 times produced little band depth changes and the NIR reflectance above $1.2 \mu \mathrm{m}$ even slightly increased as compared with irradiation with $25 \mathrm{~mJ}$ for 1 time. Repeated experimentations show that although the visible albedo at this irradiation level does decrease, the NIR albedo is very close to sample irradiated with $25 \mathrm{~mJ}$ for 1 time. It is likely that when the irradiation levels are low, the darkening effect in the NIR is not significant.

In contrast to the VNIR spectra, nearly all major MIR spectral features are retained as shown in Fig. 9, even after the heaviest irradiations. The sharp peak centered around $3 \mu \mathrm{m}$ in Fig. 9a was caused by the absorption of the reference gold mirror. The CF of the original sample around $9.0 \mu \mathrm{m}$ only slightly darkens without appreciable widening after irradiations with $25 \mathrm{~mJ} /$ pulse for 1 to 5 times, while irradiation with $50 \mathrm{~mJ} /$ pulse further broadens the CF peak. Even after the heaviest irradiation (50 $\mathrm{mJ} \times 5$ times), the CF position of olivine only slightly shifted to longer wavelength. The central wavelength of the TF between $12-15 \mu \mathrm{m}$ only shifted to the shorter wavelength by $\sim 0.13 \mu \mathrm{m}$ $\left(8 \mathrm{~cm}^{-1}\right)$ after irradiation with $50 \mathrm{~mJ} /$ pulse for 5 times, whereas the change is negligible for olivines irradiated with $25 \mathrm{~mJ} /$ pulse for 1-5 times. The restrahlen bands of olivine are weakened by the irradiation, but all the vibrational modes are identifiable after irradiations. More discussions on MIR features are deferred to Sect. 4.
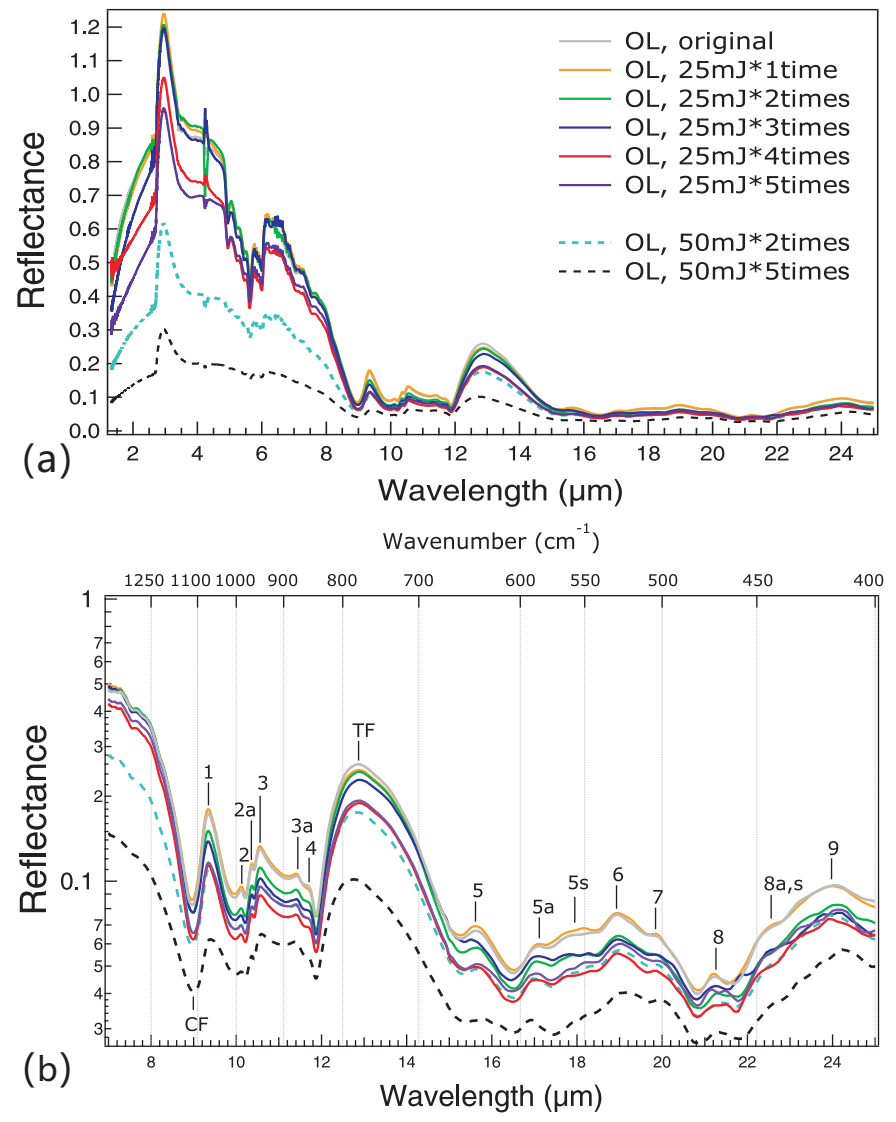

Fig. 9. MIR reflectance spectra of olivine samples before and after irradiations in decimal scale a) and logarithmic scale b). The MIR spectra were measured relative to a gold mirror stardard and then scaled to the VNIR value in the overlapping region. CF: the Christiansen Feature; TF: the Transparency Feature. The numbers indicating the vibrational modes are summarized in Table 3 in Hamilton (2010).

TEM and EDS analysis. Figure 10 shows the bright field TEM images of olivines irradiated under $50 \mathrm{~mJ}$ for 2 times. The presence of spherical particles with diameters less than $500 \mathrm{~nm}$ present on the surface of the large olivine particle (Fig. 10a) indicate that melting and redeposition processes may have occurred during the irradiations. We selected two regions of interest (ROI) as indicated by the white boxes shown in Fig. 10a to study their structures and compositions. Figure $10 \mathrm{~b}$ is the enlarged image of ROI 1 with many nanophase particles $(5-20 \mathrm{~nm})$ uniformly distributed near the surface. On the rim of ROI 2 we can find similar dark nanophase particles (Fig. 10c) smaller than $10 \mathrm{~nm}$. In order to check if the nanophase particles are $\mathrm{npFe}^{0}$ or not, we performed EDS mapping of some major elements. Figure 11a is the enlarged dark field TEM image of ROI 1 with 


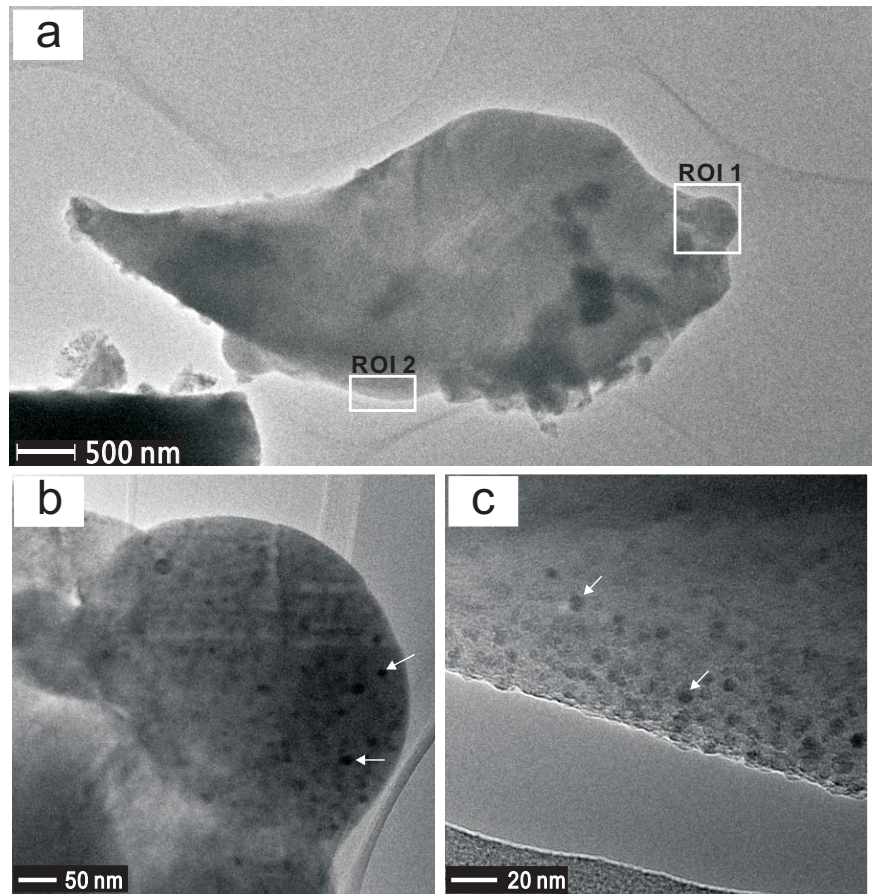

Fig. 10. Bright field TEM images of olivine samples after being irradiated under $50 \mathrm{~mJ} /$ pulse for 2 times. a) One single olivine particle with two ROIs indicated by the white boxes; b) and c) are the enlarged images of ROI 1 and ROI 2, respectively. The white arrows in b) and c) indicate the presence of nanophase particles.

the elemental mapping area indicated by the orange box. In contrast to the bright field image (Fig. 10), the nanophase particles look bright in the dark field image (Fig. 11a). As shown in Fig. $11 \mathrm{~b}$, elements $\mathrm{Si}, \mathrm{O}$ and $\mathrm{Mg}$ are uniformly distributed in the mapping area, while Fe shows an abnormal distribution feature. The Fe-rich spots are consistent with those nanophase particles shown in Fig. 10b and hence the nanophase particles are identified as $n p F e^{0}$. From the TEM images of olivines irradiated with $50 \mathrm{~mJ} /$ pulse for 2 times, we found that some olivine particles are covered by an amorphous layer. Figure 12 shows the bright field TEM images of one typical olivine particle with a $\sim 10 \mathrm{~nm}$ thick layer. In the high resolution TEM image (Fig. 12b), the inner part of the particle show clear lattice fringes, while the layer does not. This does not imply, however, that $10 \mathrm{~nm}$ is the average or maximum thickness of the produced amorphous layers in our experiments.

Figure 13 shows the TEM images and EDS mapping results of a typical olivine sample irradiated under $50 \mathrm{~mJ} /$ pulse for 5 times, the heaviest irradiation dose in this study. The diameters of the $\mathrm{nFe}^{0}$ range from 5 to $30 \mathrm{~nm}$, about $10 \mathrm{~nm}$ larger than those in the sample irradiated under $50 \mathrm{~mJ} /$ pulse 2 times.

For irradiations at lower energy levels $(25 \mathrm{~mJ})$, the $\mathrm{nFFe}^{0}$ particles produced have lower concentrations than that irradiated at $50 \mathrm{~mJ}$ and are more difficult to be found in TEM observations. However, the size of the iron grains produced is about the same as that produced by irradiations with $50 \mathrm{~mJ}$. Figure 14 compares the TEM images of olivine grains irradiated with $25 \mathrm{~mJ}$ and $50 \mathrm{~mJ}$ for 2 times, respectively. It is seen that the $\mathrm{npFe}^{0}$ grains have similar size distributions and are all smaller than $30 \mathrm{~nm}$.

XRD analysis. Figure 15 shows the XRD results of three typical olivine samples. The positions of all main diffraction peaks

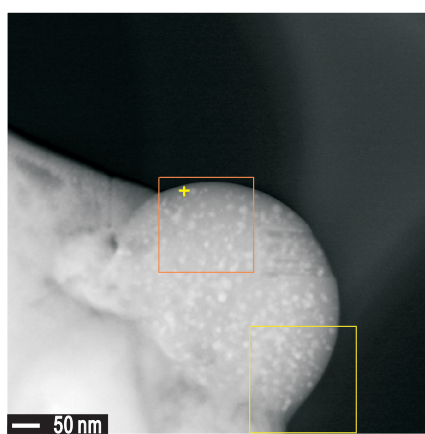

(a)

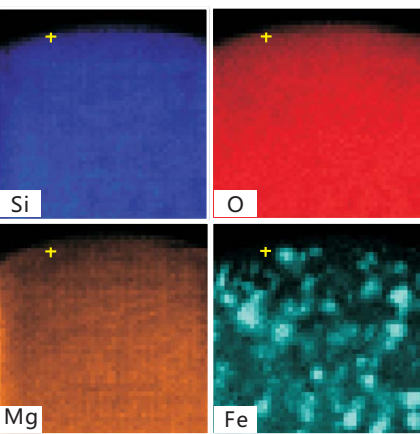

(b)
Fig. 11. EDS mapping of major elements $\mathrm{Si}, \mathrm{O}, \mathrm{Mg}$ and Fe. a) Dark field image of the ROI 1 in Fig. 10a with the orange box indicating the EDS mapping region. The area surrounded by the yellow box is used for drift corrections; b) EDS mapping results of $\mathrm{Si}, \mathrm{O}, \mathrm{Mg}$ and $\mathrm{Fe}$. The brightness of the pixels is correlated with the relative abundance of the corresponding element. The brighter spots in the Fe distribution map are consistent with the white spots in the enclosed area of the orange box, showing taht the nanoparticles are indeed nanophase irons.

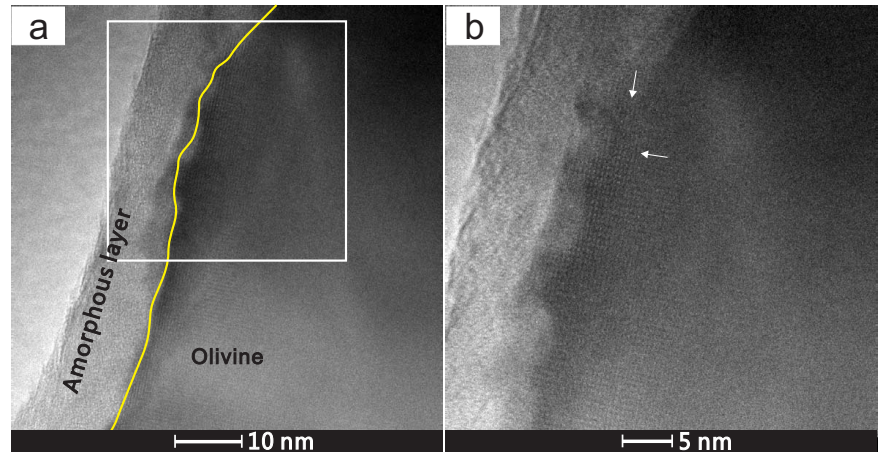

Fig. 12. Typical bright field TEM images of the olivine sample after irradiation with $50 \mathrm{~mJ} /$ pulse for 2 times. a) A yellow curve indicating the boundary between the amorphous layer and the olivine crystal; b) the high resolution image of the enclosed area of the white box in a), showing the olivine lattice fringes indicated by the white arrows.

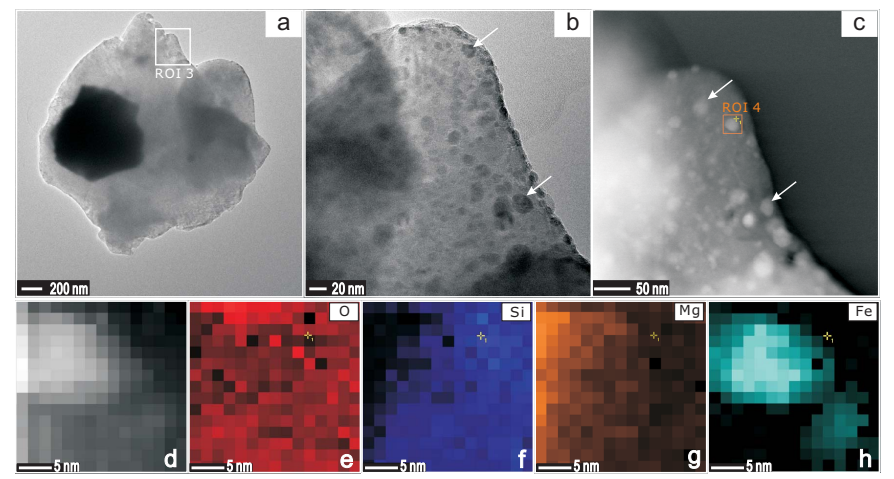

Fig. 13. TEM image and EDS mapping results of the olivine sample irradiated under $50 \mathrm{~mJ}$ for 5 times. a) Bright field image of one olivine particle; b) the enlarged image of ROI 3 as indicated by the white box in a); c) the dark field image of ROI 3, and ROI 4 is the region selected for EDS mapping; d) the high resolution image of ROI 4; e)-h) EDS mapping results of four major elements: $\mathrm{O}, \mathrm{Si}, \mathrm{Mg}$, and $\mathrm{Fe}$.

determined by the atomic distance within the mineral structure remain unchanged even after the heaviest irradiation with $50 \mathrm{~mJ} /$ pulse for 5 times. Phase analysis demonstrates that no obvious phase changes were incurred by irradiations. Although 


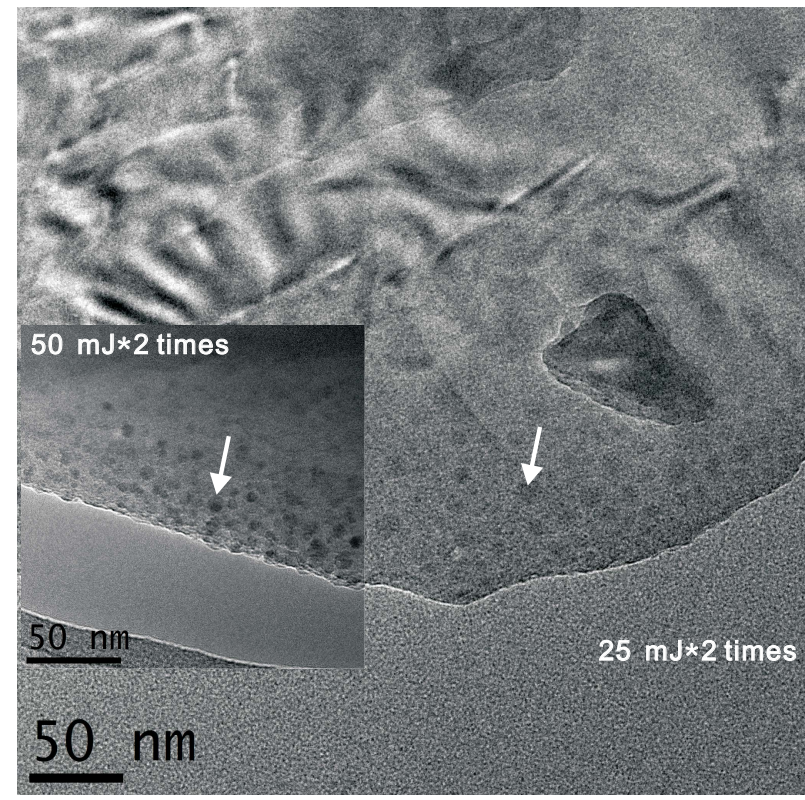

Fig. 14. TEM images of the olivine grains irradiated at $25 \mathrm{~mJ}$ for 2 times and at $50 \mathrm{~mJ}$ for 2 times (inset). The dark spots indicated by the white arrows are identified as iron particles. It is evident from this comparison that the $n \mathrm{pFe}^{0}$ particles produced at different energy levels in this work have similar size distributions $(<30 \mathrm{~nm})$.

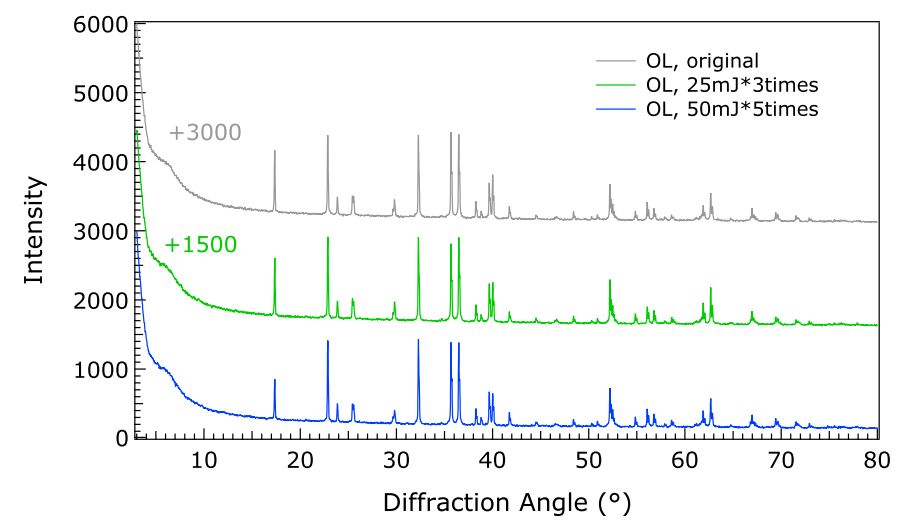

Fig. 15. XRD spectra of three typical olivine samples: the original olivine, olivine irradiated under $25 \mathrm{~mJ} /$ pulse for 3 times and olivine irradiated under $50 \mathrm{~mJ} /$ pulse for 5 times, respectively. The first two spectra are offset by an amount indicated by the numbers in graph for clarity.

amorphous layers are identified on the surfaces of irradiated olivine particles from TEM images, the XRD spectra did not show any detectable crystal structural changes.

Raman spectra. Figure 16 shows the typical Raman spectra measured under an excitation power of $9 \mathrm{~mW}$. We have found that at this power the spectra have enough signal-to-noise ratios while no heating damage was incurred during measurements. The overall spectra are shown in Fig. 16a and the enlarged weaker bands are displayed in Fig. 16b with the intensities shifted upwards for clarity. Obviously, vibrational Raman modes have survived even in the most heavily irradiated samples and thus agree with the MIR and XRD results. More discussions on Raman features are presented in Sect. 4.3.
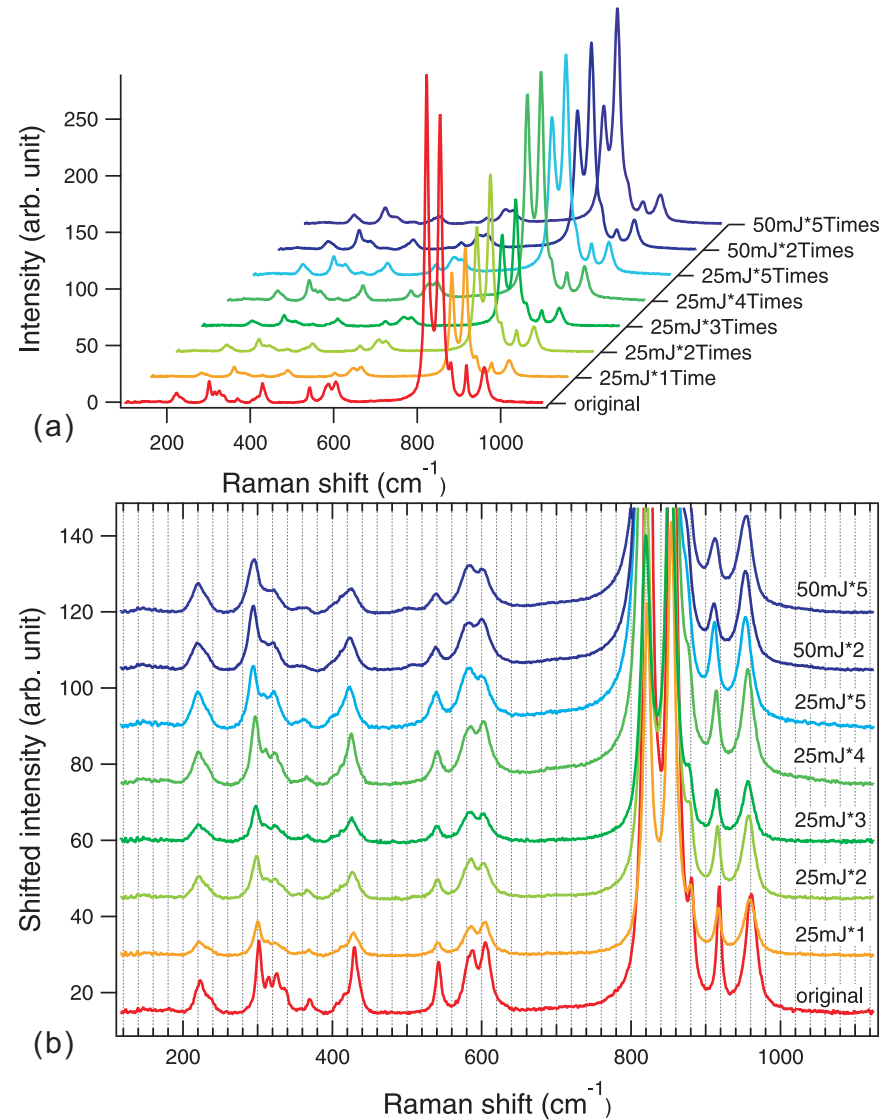

Fig. 16. Raman spectra of the original and the irradiated olivine samples. a) Waterfall plot of the sample spectra bands; b) the weaker bands that are shifted upwards for clarity.

\subsection{Results of model predictions}

The effects of $\mathrm{npFe}^{0}$ on reflectance spectra. Since the irradiation-produced $\mathrm{nFe}^{0}$ grains may either form a layer coating the particles or exist as scatters inside, their effects on optical properties need to be considered individually. For the first case, Fig. 17 shows model reflectance predictions of olivine covered by an $\mathrm{npFe}^{0}$ coating with thickness varied from 0 to $1000 \mathrm{~nm}$. The content of $\mathrm{npFe}^{0}$ within the coating was set to a very high value of 1 vol. \%. Both the Hapke model (Fig. 17a) and the DISORT (Fig. 17b) predictions show that the existence of $n \mathrm{pFe}^{0}$ result in an overall reduced reflectance, subdued absorption features, and a reddened VNIR spectra consistent with the previous pulsed laser irradiation experiments (e.g., Yamada et al. 1999; Sasaki et al. 2003). However, the MIR spectra remain essentially unchanged even when the $n \mathrm{pre}^{0}$ coating thickness reaches $1000 \mathrm{~nm}$. For the second case, Fig. 18 shows the modeled reflectance of $\mathrm{npFe}^{0}$ uniformly distributed inside olivine grains (inset of Fig. 18a). As shown by both the Hapke model and the DISORT predictions, an amount of $0.01 \mathrm{vol} . \% \mathrm{npFe}^{0}$ is sufficient to cause appreciable feature changes in the VNIR spectra, while the MIR bands almost remain unchanged even when the $\mathrm{npFe}^{0}$ contents reaches an unrealistically high level of 1 vol. \% (Morris 1980).

The effects of amorphous layer. We next consider the effects of an amorphous layer coating the olivine particles by fixing the diameter of an olivine core of $30 \mu \mathrm{m}$ and varying the coating thickness from $0 \mathrm{~nm}$ to $1000 \mathrm{~nm}$. In addition to layer thickness 

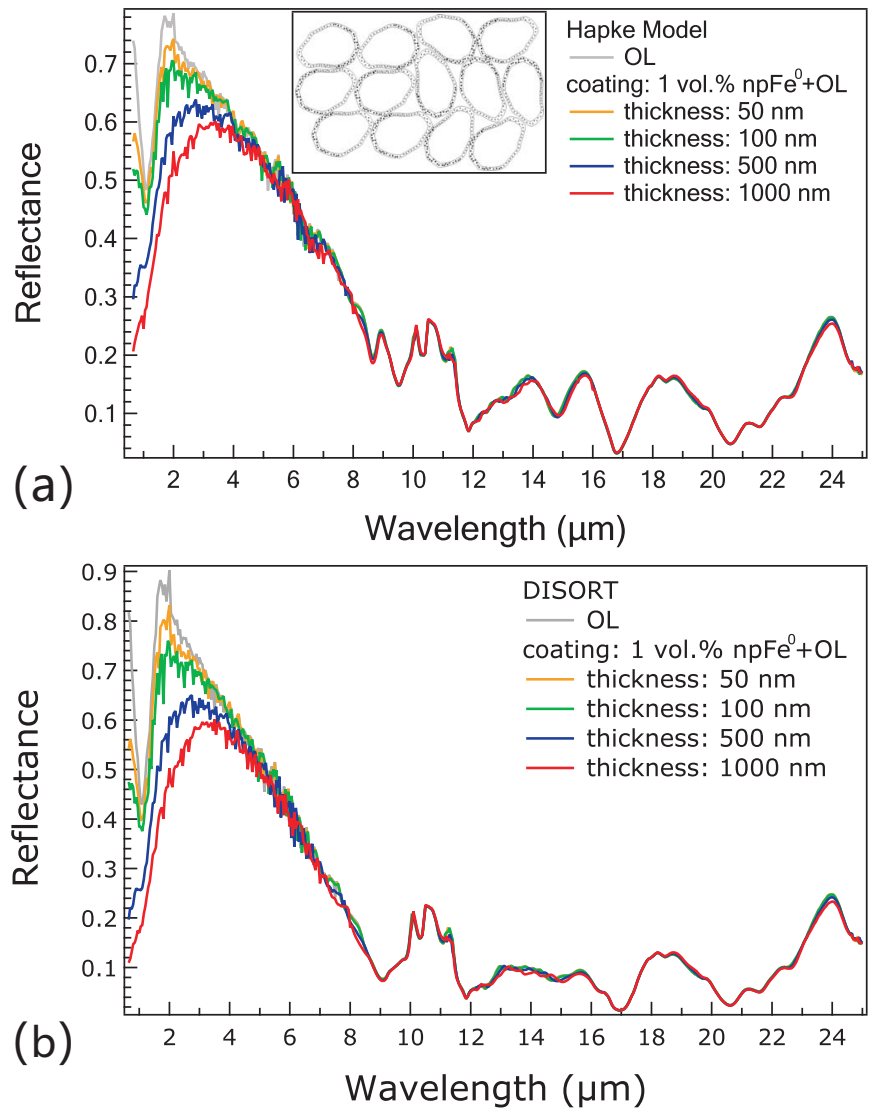

Fig. 17. Modeled reflectance of olivines with various thicknesses of npFe ${ }^{0}$ coatings. a) Results of the Hapke Model; b) results of the DISORT. The inset in a) is a schematic showing the $\mathrm{nFFe}^{0}$ distribution patterns used in modelings. The content of $\mathrm{npFe}^{0}$ in the coatings was set to be 1 vol. $\%$.

effects, we also looked at the effects of four different compositions of the glassy coatings (see Sect. 2.2).

Figure 19 shows the reflectance predictions of olivine coated by Glass $\left.1\left(\mathrm{Fo}_{95}\right)\right)$. The modeled results indicate that the MIR spectra may be affected by glassy coating. The vibrational bands are weakened as the coating thickness increases, which is consistent with the MIR measurements on laser irradiated olivine particles. Although differences of CF positions exist between the two model predictions (Hapke model: $8.65 \mu \mathrm{m}$; DISORT: $9.05 \mu \mathrm{m}$ ), both models predict that the CF position slightly shifted $(0.05 \mu \mathrm{m})$ to shorter wavelength when the coating is thick enough $(>500 \mathrm{~nm})$. However, the shift is negligible when the layer thickness is smaller than $200 \mathrm{~nm}$ which is larger than the upper limit of the layers among the particle samples returned from Itokawa (Noguchi et al. 2011). Figure 20 shows the modeled spectra of olivine particles coated with Glass 2 which contains more Fe compared to Glass 1 . Both models show that more Fe within the glassy coating darkens and reddens the VNIR spectra, similar to the effects produced by the $n p \mathrm{Fe}^{0}$. However, the MIR spectral feature changes incurred from a different $\mathrm{Fe}$ content (Glass 2 vs. Glass 1) are negligible as compared with the NIR feature changes. Figures 21 and 22 show the predicted spectra of olivine particles coated with Glass 3 and Glass 4, respectively. Compared with pyroxene-type glassy coatings (Glass 1 and Glass 2), olivine-type glass coatings (Glass 3 and Glass 4) make the CF position of olivine shift towards longer wavelength which is more consistent with our measurements. This may indicate that the glassy coating produced in our simulations are
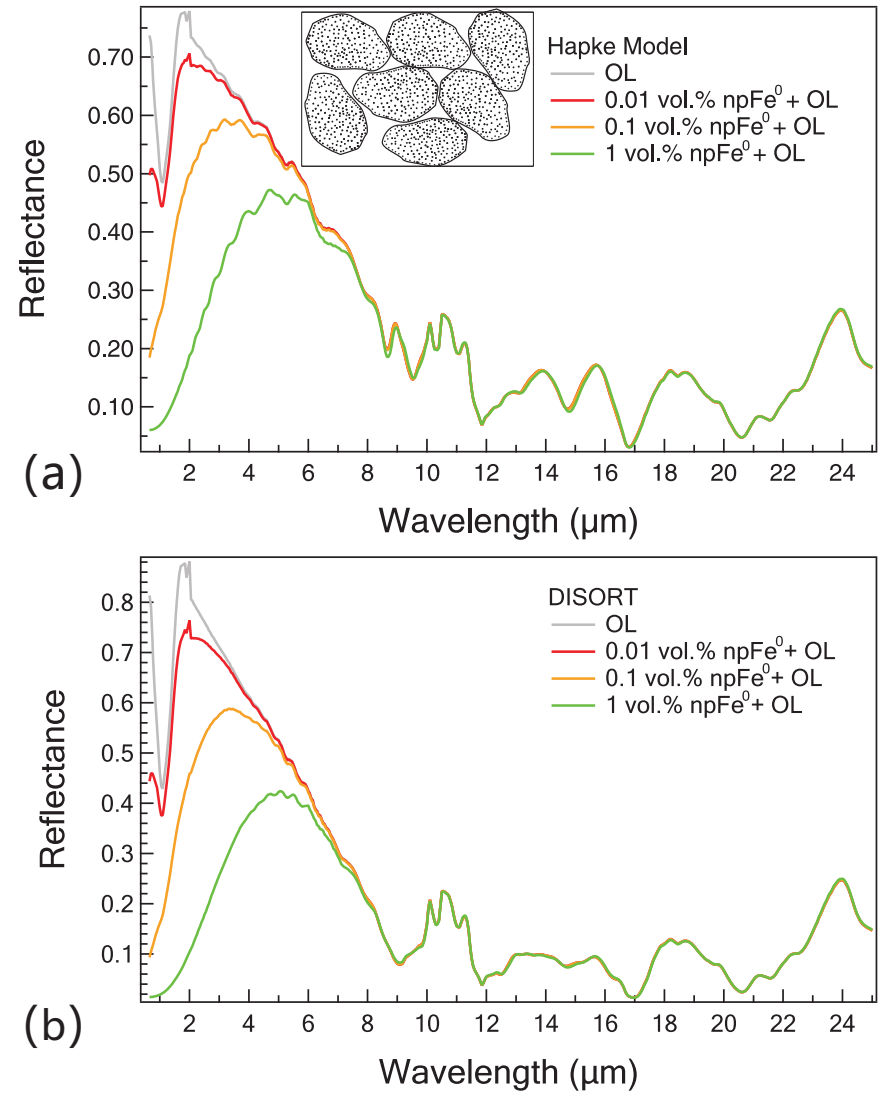

Fig. 18. Modeled reflectance of olivines with various contents of $n p F e^{0}$. a) Results of the Hapke Model; b) results of the DISORT. The $n p \mathrm{Fe}^{0}$ is assumed to be evenly distributed in the grains as indicated by the inset in a).

compositionally similar to olivine-type glass. Except for this difference, all four different glassy coatings caused similar darkening effects on the MIR spectra of olivine grains.

The broad peaks appearing between 2 and $8 \mu \mathrm{m}$ in model predictions can be grouped into two categories: those redshift (Figs. 17, 18, 20 and 22) and those remain unshifted (Figs. 19 and 21) as glassy coating thickness or iron content increases. Specifically, redshifts and no-shifts occur in iron-rich and ironpoor environments, respectively. Unfortunately, the measured spectra in this region (Fig. 9a) are contaminated by the strong absorption of the reference gold mirror near $3 \mu \mathrm{m}$ which prevents a decisive model/measurement comparison. Future work on this broad NIR feature is expected.

\section{Discussions}

\subsection{VNIR spectral features}

Our pulsed laser irradiation experiments have successfully reproduced the optical effects of space weathering in the VNIR region which are consistent with previous simulation results (e.g., Yamada et al. 1999; Sasaki et al. 2003). By performing TEM and EDS analysis, we identified two types of $\mathrm{nFFe}^{0}$, one contained in the melted spherical particles (Fig. 10a) and the other one distributed in the rim of some olivine particles. These morphologies are very similar to the $n p F e^{0}$ distributions in the returned lunar and Itokawa samples (e.g., Keller \& McKay 1997; Noguchi et al. 2011). Most of the $n p F e^{0}$ particles produced in our experiments are smaller than $10 \mathrm{~nm}$ in diameter. Although some $\mathrm{nFe}^{0}$ particles can reach $30 \mathrm{~nm}$ in diameter in 

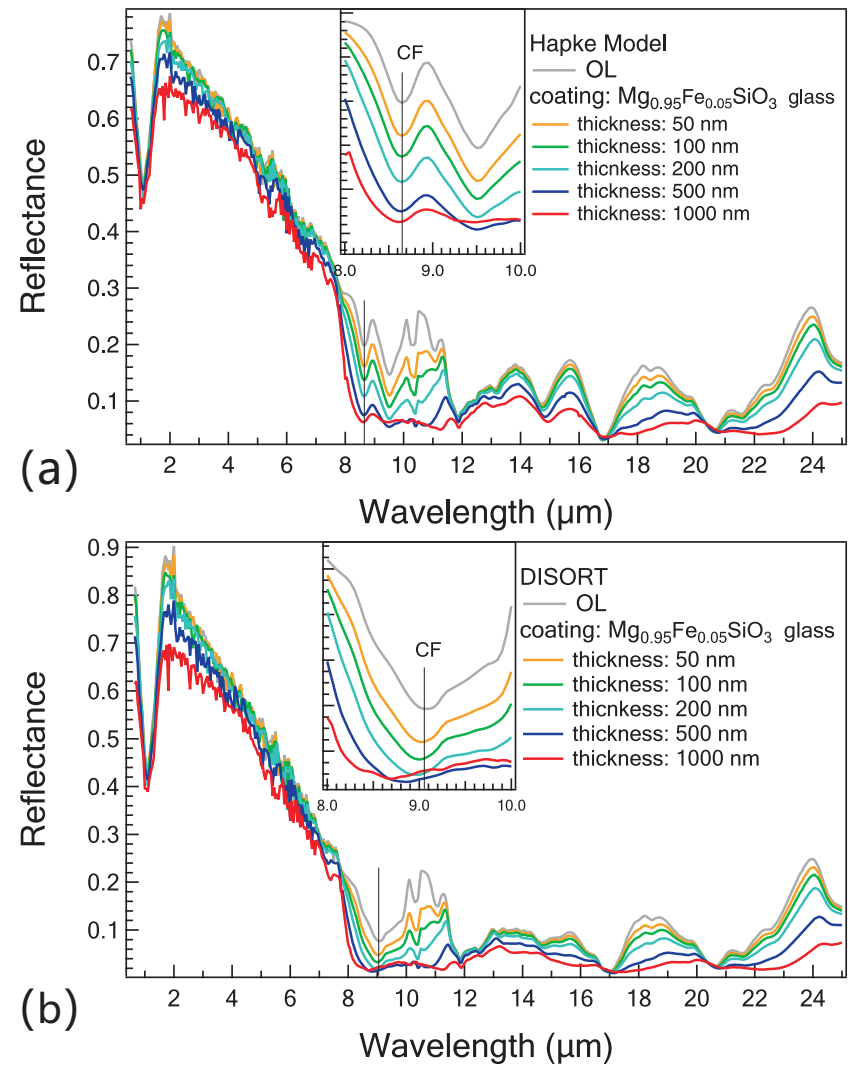

Fig. 19. Modeled reflectance of olivines coated by Glass 1. Predictions of the Hapke model a) and the DISORT b). The insets are enlarged $\mathrm{CF}$ features and the vertical black lines indicate the CF positions of the uncoated olivine.
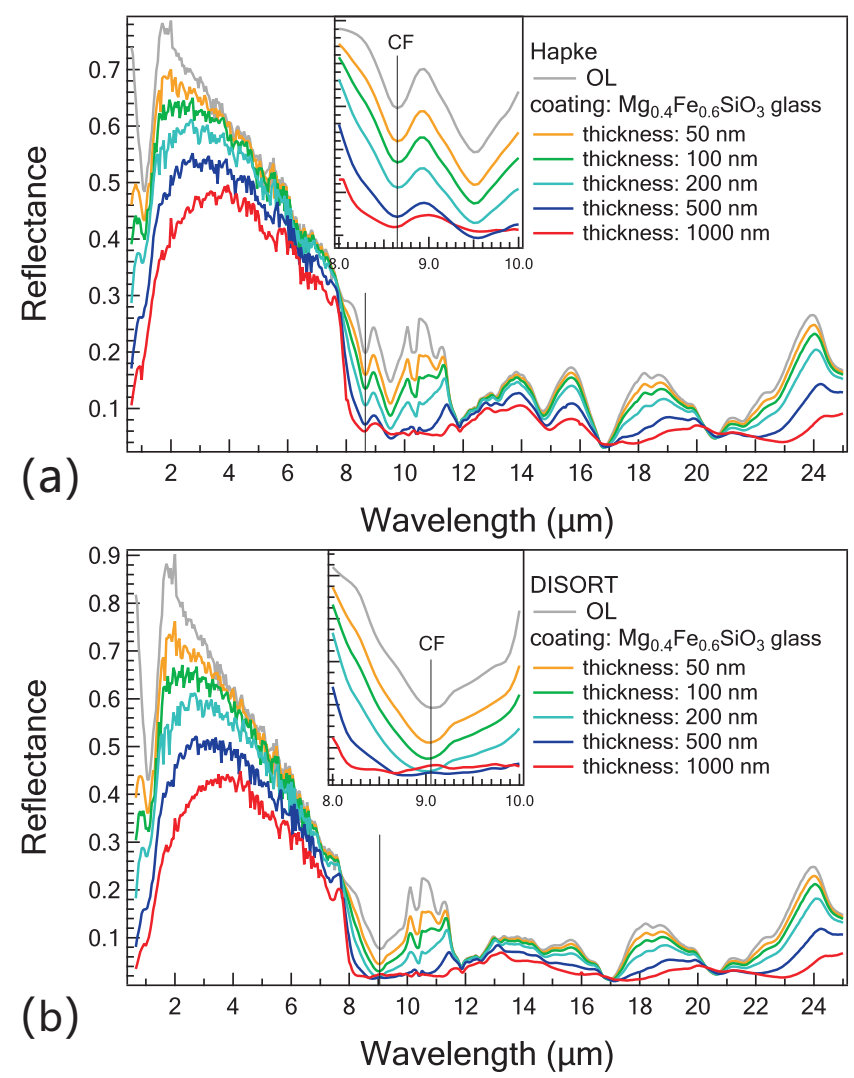

Fig. 20. Same as Fig. 19 but coated by Glass 2 .
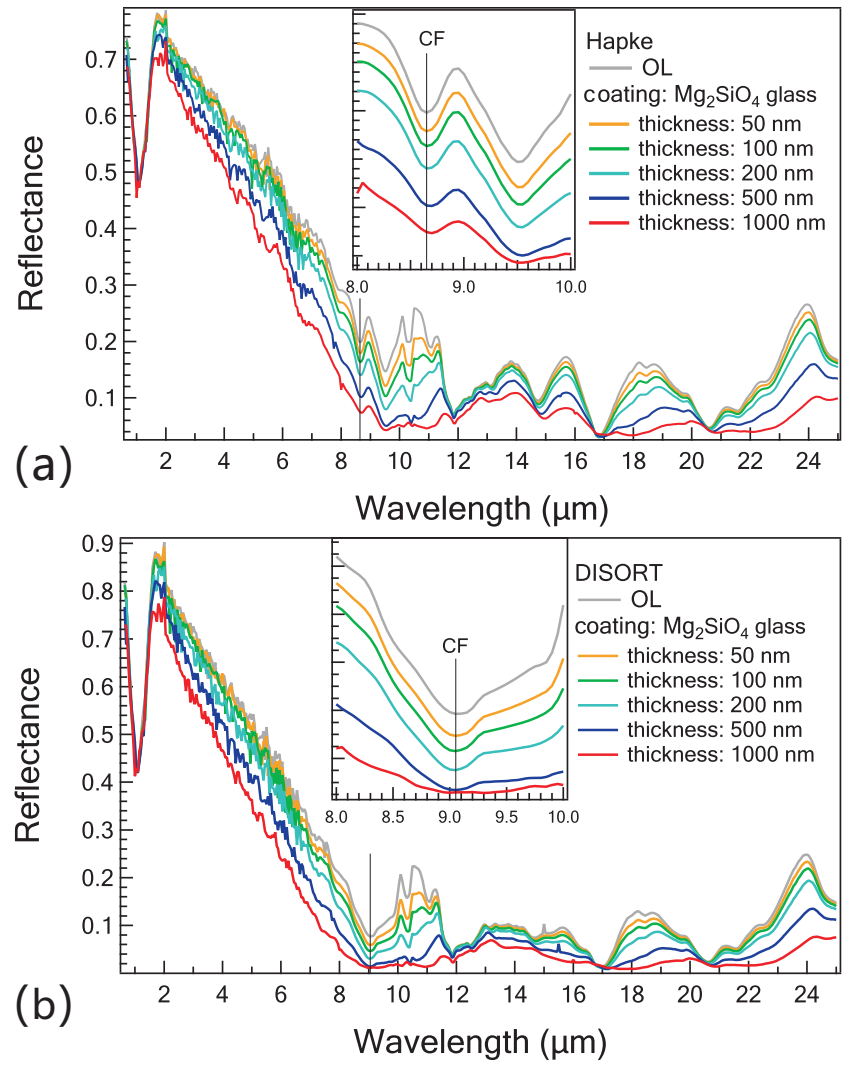

Fig. 21. Same as Fig. 19 but coated by Glass 3 .
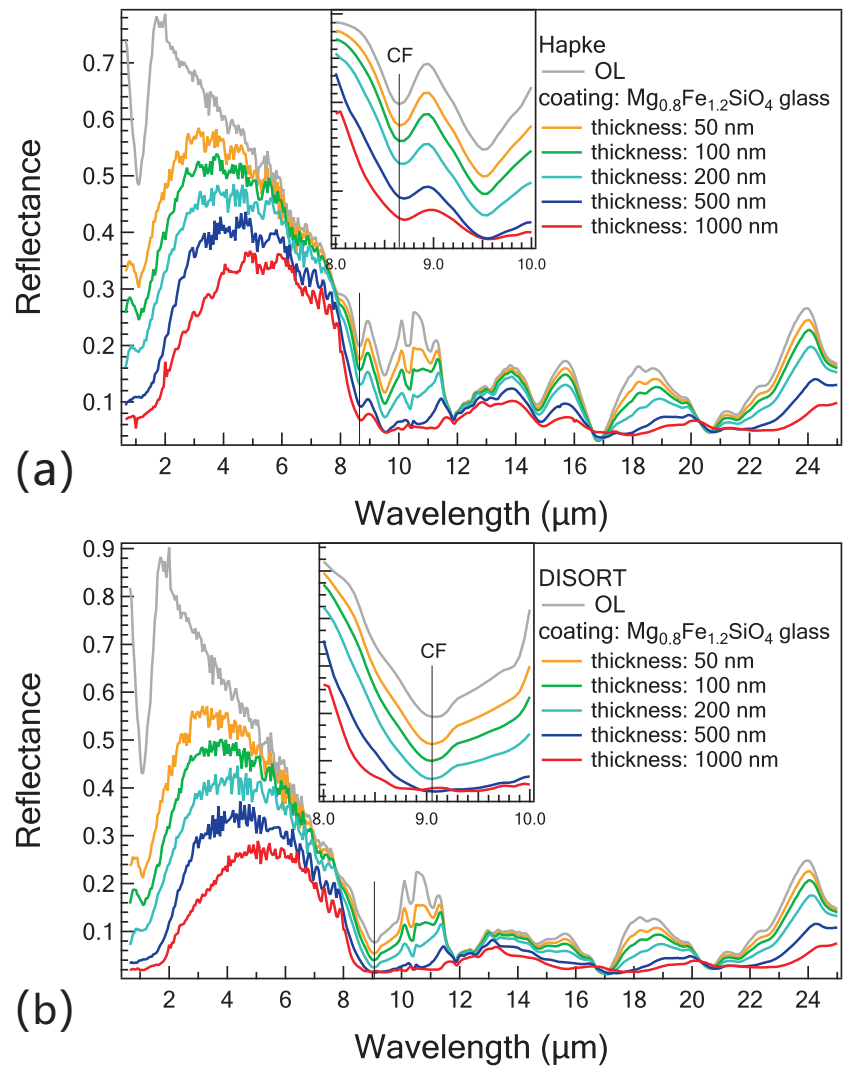

Fig. 22. Same as Fig. 19 but coated by Glass 4 . 
the aggregates after irradiation with $50 \mathrm{~mJ} /$ pulse for 5 times, they are still considerably smaller than the $\mathrm{npFe}^{0}$ found in returned lunar agglutinates (e.g., Pieters et al. 2000).

For the $25 \mathrm{~mJ} /$ pulse irradiation, our results indicate that the changes of the $1 \mu \mathrm{m}$ band depth are proportional to number of irradiations, while Sasaki et al. (2003) shows that the spectral change could be saturated after being irradiated under $30 \mathrm{~mJ} /$ pulse for 5 times. Compared with Sasaki et al. (2003), we set a much slower scanning velocity $\left(1 \mathrm{~mm} \mathrm{~s}^{-1}\right)$ compared to their $5 \mathrm{~mm} \mathrm{~s}^{-1}$. As a result, the imparted energy of our one irradiation experiment in unit area is $750 \mathrm{~mJ} / \mathrm{mm}^{2}$ for $25 \mathrm{~mJ} /$ pulse which is much larger than their $240 \mathrm{~mJ} / \mathrm{mm}^{2}$ for $30 \mathrm{~mJ} / \mathrm{pulse}$. In terms of the total deposited energy, their five-time irradiation under $30 \mathrm{~mJ} /$ pulse is only equivalent to two-time irradiation under $25 \mathrm{~mJ} /$ pulse in our experiment. However, we did not find the similar saturation trend. Considering that the difference of the energy per pulse is small, the major difference between our results should be caused by the particle packing conditions, as Sasaki et al. (2003) irradiated packed grains in pellet form while we used loose powders. Thus it is likely that a compact surface would be more easily to be saturated in spectra changes. Therefore, the surface structure and laser scanning velocity must be carefully considered if we want to evaluate the real weathering duration through pulsed laser simulation experiments.

The above results imply that the weathering degree, as manifest by the darkening, reddening, and the subdued $1 \mu \mathrm{m}$ absorption features, is not simply controlled by the total deposited energy, but also the single pulse energy. With increased number of irradiations, the spectral features tend to disappear (Fig. 5) and the overall spectrum approaches the continuum (Fig. 7a). However, when the sample irradiated with $25 \mathrm{~mJ}$ for 5 times $\left(3750 \mathrm{~mJ} / \mathrm{mm}^{2}\right.$ total deposited energy) is compared to that irradiated with $50 \mathrm{~mJ}$ for 2 times $\left(3000 \mathrm{~mJ} / \mathrm{mm}^{2}\right.$ total deposited energy), the latter one is found to have more significant darkening and more subdued spectral features but less reddening feature, though the latter one's total deposited energy is $750 \mathrm{~mJ} / \mathrm{mm}^{2}$ less. We have performed repeated experimentations to make sure the above variation trends can be reproduced reliably. This may indicate that spectral reddening is more susceptible to energy deposition rate (single pulse energy level) and could be explained by the argument that irradiations at higher energy deposition rate might produce larger $\mathrm{npFe}^{0}$ grains $(>50 \mathrm{~nm})$ that darken but not redden the spectra (Lucey \& Riner 2011). However, the comparisons shown in Fig. 14 clearly demonstrate that the $\mathrm{npFe}^{0}$ grains produced at $50 \mathrm{~mJ} /$ pulse and $25 \mathrm{~mJ} /$ pulse in our simulations have commensurate size and are much smaller than $50 \mathrm{~nm}$. Therefore, a more plausible explanation is that at higher energy deposition rate the weathered grains are coated by thicker glassy layers and thus any $\mathrm{npFe}^{0}$ induced reddening is greatly suppressed by darkening. Although we are unable to determine the actual layer thickness produced by $50 \mathrm{~mJ} /$ pulse irradiations, model predictions as shown in Fig. 22 clearly show that an iron-rich olivine-type glassy coating can significantly darken the VNIR spectra.

In the current study we ignored any possible $\mathrm{nFFe}^{0}$ grain size effects on reflectance and this is a major shortcoming especially for VNIR model predictions. Although a modeling approach proposed by Lucey \& Riner (2011) has accounted for the $n p F e^{0}$ particle size effect, it is only applicable to VNIR spectra as in this region the single scattering phase function can be approximated by a constant value. Moreover, Lucey \& Riner (2011) found that the $n p \mathrm{Fe}^{0}$ grain size effects on modeling results are only significant at short wavelengths. As we have pointed out, we concentrate our model/measurement comparisons on MIR regions where the radiation wavelength is much larger than the grain size of the $\mathrm{nFF}^{0}$ particles produced in our simulations $(<30 \mathrm{~nm})$, and thus ignoring the $n \mathrm{pFe}^{0}$ size should not incur serious errors. The $n \mathrm{pFe}^{0}$ grain size effects on VNIR spectra merit further investigations with controlled production of $n \mathrm{pFe}^{0}$ with various size distributions.

\subsection{MIR reflectance features}

Group theoretical analysis yields totally 35 infrared-active vibrational modes for olivine crystal as $13 \mathrm{~B}_{1 u}+9 \mathrm{~B}_{2 u}+13 \mathrm{~B}_{3 u}$ (e.g., Paques-Ledent \& Tarte 1973; Hofmeister 1987). Although mode assignment can be made by performing polarized reflectance measurements on aligned single crystals, it would be very difficult to identify the mode symmetry for each band in randomly orientated small grains as the $\mathrm{B}_{1 u}, \mathrm{~B}_{2 u}$ and $\mathrm{B}_{3 u}$ modes appear in overlapping spectral regions and have similar spectral shapes (Hofmeister 1987). As a result, usually a band number instead of a specific irreducible representation is assigned to each distinct reflectance peak appearing in the MIR region of powdered samples. Comparisons of our measurement on unirradiated olivine sample with previous measurements (as summarized in Table 3 in Hamilton 2010) and lattice dynamics computations (Noel et al. 2006) give the following identifications (in $\mathrm{cm}^{-1}$ ): 1070 (band 1), 988 (band 2), 964 (band 2a), 947 (band 3), 876 (band 3a), 853 (band 4, s), 777 (TF), 640 (band 5), 584 (band 5a), 557-546 (band 5s), 528 (band 6), 503 (band 7), 471 (band 8), 442 (band 8a, s), 417 (band 9) (s as shoulder). Table 3 summarizes the identifications in both wavenumbers and microns. Among these peaks, bands 1 to 4 and bands 5 to $8 \mathrm{a}$ belong to the stretching and bending of the $\mathrm{SiO}_{4}$ group, respectively (Noel et al. 2006). Band 9 either belongs to relative motions between $\mathrm{Fe}$ and $\mathrm{SiO}_{4}$ or $\mathrm{SiO}_{4}$ bending (Noel et al. 2006). Nearly all bands have survived the heaviest irradiations $(50 \mathrm{~mJ} /$ pulse for 5 times $)$, as can be seen in Fig. 9. As Sect. 3.1 shows, the MIR reflectance spectral features of olivine are quite immune to the productions of $n p \mathrm{Fe}^{0}$. In the irradiated olivine particles, we have confirmed the existence of the $n p F e^{0}$ and the VNIR reflectance spectra of these olivine samples changed a lot. However, nearly all major MIR vibrational features are retained even after the heaviest irradiation process under $50 \mathrm{~mJ} /$ pulse for 5 times which may be "over-irradiated" as compared with real space weathering processes on asteroids (e.g., Clark et al. 2002; Chapman 2004).

The reflectance modeling results show that the MIR spectra of olivine are not susceptible to $n p F e^{0}$, even when the $n p F e^{0}$ content reaches an unrealistic high level of 1 vol. \% (Morris 1980). In contrast, amorphous coatings darken and weaken the vibrational bands more efficiently, as revealed by both the TEM/MIR measurements and the Hapke and the DISORT model predictions. Moreover, an olivine-type glassy coating $\left(\mathrm{Mg}_{x} \mathrm{Fe}_{2-x} \mathrm{SiO}_{4}\right)$ may shift the CF position of olivine towards longer wavelength when the coating is thick enough. However, as long as the glassy coating thickness is less than $200 \mathrm{~nm}$, which is much larger than the upper value of returned asteroid samples, the CF position and other MIR features are very well retained. It is also found that the effects of irradiations with $25 \mathrm{~mJ} /$ pulse on the MIR spectra are rather limited. This may occur because low energy deposition rate did not produce thick enough coatings.

Recently Lucey et al. (2016) found the Christiansen Feature of more space-weathered lunar surface shifts to longer wavelength as compared to less weathered area based on Diviner observations over the Moon, though the magnitude of the shift is only a few percent of one micron. Although our reflectance 
Table 3. MIR Band positions of olivines before and after irradiations in wavenumbers (upper) and microns (lower).

\begin{tabular}{|c|c|c|c|c|c|c|c|c|c|c|c|c|c|c|c|c|}
\hline $\mathrm{OL}^{*}$ & $\mathrm{CF}$ & $\begin{array}{l}\text { Band } \\
1\end{array}$ & $\begin{array}{l}\text { Band } \\
2\end{array}$ & $\begin{array}{l}\text { Band } \\
2 \mathrm{a}\end{array}$ & $\begin{array}{l}\text { Band } \\
3\end{array}$ & $\begin{array}{l}\text { Band } \\
3 a\end{array}$ & $\begin{array}{l}\text { Band } \\
4\end{array}$ & $\mathrm{TF}$ & $\begin{array}{l}\text { Band } \\
5\end{array}$ & $\begin{array}{l}\text { Band } \\
5 a\end{array}$ & $\begin{array}{l}\text { Band } \\
5 s\end{array}$ & $\begin{array}{l}\text { Band } \\
6\end{array}$ & $\begin{array}{l}\text { Band } \\
7\end{array}$ & $\begin{array}{l}\text { Band } \\
8\end{array}$ & $\begin{array}{l}\text { Band } \\
8 a, s\end{array}$ & $\begin{array}{l}\text { Band } \\
9\end{array}$ \\
\hline 0 & 1115 & 1070 & 988 & 964 & 947 & 876 & 853 & 777 & 640 & 584 & $557-546$ & 528 & 503 & 471 & 442 & 417 \\
\hline 1 & 1117 & 1070 & 988 & 964 & 947 & 878 & 853 & 775 & 640 & 584 & $557-5$ & 528 & 503 & 471 & 442 & 417 \\
\hline 2 & 1113 & 1070 & 989 & 964 & 947 & 878 & 853 & 777 & 638 & 586 & $557-546$ & 527 & 503 & 469 & 442 & 415 \\
\hline 3 & 1113 & 1070 & 989 & 964 & 947 & 876 & 851 & 775 & 644 & 584 & $557-546$ & 528 & 501 & 471 & 442 & 415 \\
\hline 4 & 1113 & 1070 & 989 & 964 & 947 & 878 & 853 & 775 & 640 & 586 & $557-546$ & 528 & 501 & 467 & 442 & 419 \\
\hline 5 & 1113 & 1070 & 989 & 964 & 945 & 880 & 853 & 775 & 638 & 586 & $557-546$ & 527 & 501 & 473 & 442 & 415 \\
\hline 6 & 1115 & 1069 & 989 & 964 & 947 & 878 & 853 & 777 & 640 & 590 & & 527 & 503 & 469 & 442 & 419 \\
\hline 7 & 1109 & 1065 & 989 & 964 & 945 & 880 & 853 & 785 & 635 & 590 & $557-546$ & 523 & 501 & 469 & 442 & 413 \\
\hline $\mathrm{OL}^{*}$ & $\mathrm{CF}$ & $\begin{array}{l}\text { Band } \\
1\end{array}$ & $\begin{array}{l}\text { Band } \\
2\end{array}$ & $\begin{array}{l}\text { Band } \\
2 \mathrm{a}\end{array}$ & $\begin{array}{l}\text { Band } \\
3\end{array}$ & $\begin{array}{l}\text { Band } \\
3 a\end{array}$ & $\begin{array}{l}\text { Band } \\
4\end{array}$ & $\mathrm{TF}$ & $\begin{array}{l}\text { Band } \\
5\end{array}$ & $\begin{array}{l}\text { Band } \\
5 a\end{array}$ & $\begin{array}{l}\text { Band } \\
5 \mathrm{~s}\end{array}$ & $\begin{array}{l}\text { Band } \\
6\end{array}$ & $\begin{array}{l}\text { Band } \\
7\end{array}$ & $\begin{array}{l}\text { Band } \\
8\end{array}$ & & $\begin{array}{l}\text { Band } \\
9\end{array}$ \\
\hline 0 & 8.97 & 9.34 & 10.13 & 10.37 & 10.56 & 11.42 & 11.73 & 12.87 & 15.62 & 17.11 & 17.94 & 18.92 & 19.87 & 21.25 & 22.542 & 24.00 \\
\hline 1 & 95 & 9.34 & 13 & 10.37 & 10.56 & 11.40 & 11.73 & 12.90 & 15.6 & 17.11 & 17.94 & 92 & 19.87 & 21.25 & & 4.00 \\
\hline 2 & 99 & 9.34 & .11 & 10.37 & 10.56 & 11.40 & 11.73 & 12.87 & 15.6 & 17.06 & 17.94 & 99 & 19.87 & 21.34 & & 24.12 \\
\hline 3 & 99 & 9.34 & 0.11 & 10.37 & 10.56 & 11.42 & 11.76 & 12.90 & 15.52 & 17.11 & 17.94 & 18.92 & 19.94 & 21.25 & 22.542 & 24.12 \\
\hline 4 & & 9.34 & & & & 11.40 & 11.73 & 12. & 15.62 & 17.06 & 17. & & & 21.42 & & 23.89 \\
\hline 5 & 99 & 9.34 & 1 & & 10. & 11.37 & 11.73 & 12.90 & 15.66 & 17.06 & 17.94 & 18 & 19.94 & 21.16 & 22.542 & 24.12 \\
\hline 6 & & 9.36 & 10.11 & 10.37 & 10.56 & 11.40 & 11.73 & 12.87 & 15.62 & 16.94 & 17.94 & 18.99 & 19.87 & 21.34 & 22.542 & 23.89 \\
\hline 7 & 9.02 & 9.39 & 10.11 & 10.37 & 10.58 & 11.37 & 11.73 & 12.74 & 15.76 & 16.94 & 17.94 & 19.13 & 19.94 & 21.34 & 22.542 & 24.23 \\
\hline
\end{tabular}

Notes. ${ }^{(*)}$ The numbers indicate the olivines with different irradiation degrees: 0 (unirradiated); $1(25 \mathrm{~mJ} /$ pulse $\times 1$ time $) ; 2(25 \mathrm{~mJ} /$ pulse $\times 2$ times); $3(25 \mathrm{~mJ} /$ pulse $\times 3$ times $) ; 4(25 \mathrm{~mJ} /$ pulse $\times 4$ times $) ; 5(25 \mathrm{~mJ} /$ pulse $\times 5$ times $) ; 6(50 \mathrm{~mJ} /$ pulse $\times 2$ times $) ; 7(50 \mathrm{~mJ} /$ pulse $\times 5$ times $)$

Table 4. Raman mode assignments of the olivine samples based on Kolesov \& Geiger (2004).

\begin{tabular}{|c|c|c|c|c|c|c|c|c|c|c|c|c|c|c|c|}
\hline $\mathrm{OL}^{*}$ & $\mathrm{Ag}$ & B3g & $\mathrm{B} 2 \mathrm{~g}$ & $\mathrm{Ag}$ & $\mathrm{Ag}$ & $\mathrm{Ag}$ & $\mathrm{B} 1 \mathrm{~g}+\mathrm{B} 2 \mathrm{~g}+\mathrm{B} 3 \mathrm{~g}$ & $\mathrm{Ag}$ & $\mathrm{B} 1 \mathrm{~g}+\mathrm{B} 2 \mathrm{~g}$ & B3g? & $\mathrm{Ag}$ & $\mathrm{Ag}$ & $\mathrm{B} 1 \mathrm{~g}+\mathrm{B} 3 \mathrm{~g}$ & $\mathrm{Ag}$ & $\mathrm{Ag}$ \\
\hline 0 & 961 & 918 & 881 & 855 & 823 & 605 & 588 & 542 & 430 & 369 & $336(s)$ & 326 & 315 & 301 & 223 \\
\hline 1 & 958 & 917 & 879 & 854 & 822 & 605 & 586 & 541 & 429 & 368 & - & 323 & 314 & 300 & 222 \\
\hline 2 & 958 & 916 & 878 & 853 & 821 & 602 & 587 & 541 & 426 & 365 & - & 323 & 314 & 298 & 222 \\
\hline 3 & 957 & 914 & $\sim 877(\mathrm{~s})^{(* *)}$ & 852 & 820 & 603 & 586 & 541 & 426 & 366 & - & 322 & $\sim 311$ & 298 & 220 \\
\hline 4 & 956 & 914 & $\sim 875(\mathrm{~s})$ & 852 & 819 & 602 & 585 & 541 & 426 & 365 & - & 323 & 311 & 297 & 220 \\
\hline 5 & 953 & 912 & $\sim 872(\mathrm{~s})$ & 851 & 817 & 599 & 584 & 539 & 423 & 361 & - & 322 & 308 & 295 & 220 \\
\hline 6 & 953 & 911 & $\sim 874(\mathrm{~s})$ & 851 & 817 & 600 & 584 & 538 & 423 & 359 & - & 321 & $\sim 308$ & 294 & 219 \\
\hline 7 & 955 & 912 & $\sim 874(\mathrm{~s})$ & 851 & 819 & 599 & 585 & 539 & 426 & 365 & - & 321 & - & 296 & 221 \\
\hline
\end{tabular}

Notes. ${ }^{(*)}$ The sample numbers are the same as those used in Table $3 ;^{(* *)}$ s: shoulder.

measurements were carried out in ambient environment, the band positions summarized in Table 3 suggest that the CF positions of the heavily irradiated samples indeed have shifted to longer wavelengths, from $8.97 \mu \mathrm{m}$ for unirradiated sample to $9.02 \mu \mathrm{m}$ for $50 \mathrm{~mJ} /$ pulse 5 times (the $4 \mathrm{~cm}^{-1}$ spectral resolution in the MIR region used in our measurements translates to $0.04 \mu \mathrm{m}$ in wavelength scale). For bands other than the $\mathrm{CF}$ and the TF, many bands exhibit a redshift of several wavenumbers upon irradiations. This is consistent with the Raman results shown in 4.3 and should be caused by the tensile strain induced mode softening (Cerdeira et al. 1972) due to the presence of lattice defects upon irradiations.

\subsection{Raman spectral features}

As summarized in Table 4, out of the total 36 vibrational Raman modes for olivine crystal, $11 \mathrm{~A}_{g}+7 \mathrm{~B}_{1 g}+11 \mathrm{~B}_{2} g+7 \mathrm{~B}_{3} g, 14$ peaks have been identified by comparing with previous powdered sample measurements (Kolesov \& Geiger 2004). Laser irradiations caused two major spectral feature changes. First, the full width at half maximum (FWHM) of all Raman peaks increased upon irradiations (Fig. 16b). Second, upon irradiations all Raman modes experienced downshifts or mode softening by $4-6 \mathrm{~cm}^{-1}$ (Table 4$)$. These two features are strong indications of the presence of lattice defects after irradiations. The increase of the FWHM should be caused by the presence of glassy (amorphous) materials produced during the irradiation. The mode softening may be caused by coatings of crystalline olivine grains composed of nanophase irons and glassy materials, as tensile strains caused by lattice defects are known to downshift the Raman peaks (Cerdeira et al. 1972). However, the crystalline Raman features are very well preserved even under the most intense irradiations. This is quite consistent with the MIR and XRD features and thus we conclude that the crystal lattice survived the strong irradiations.

\section{Conclusions}

By performing pulsed laser irradiations on olivine grains with various energy levels and time durations, we have successfully reproduced the typical space weathering-induced reddening and darkening effects in the VNIR reflectance spectra. Based on the TEM analysis, we have identified the production of $n \mathrm{pFe}^{0}$, and the iron grains are much smaller than $30 \mathrm{~nm}$.

When using pulsed laser irradiation to simulate the micrometeorite impacts, the energy level should be carefully considered. Two different energy levels, $25 \mathrm{~mJ}$ and $50 \mathrm{~mJ}$, have resulted in 
two different variation trends of the VNIR spectral features as the number of irradiations increases. Irradiations at $25 \mathrm{~mJ}$ for 1 to 5 times have produced progressively overall shallower $1 \mu \mathrm{m}$ band depth, lower albedo, and redder spectral slope. Irradiations at $50 \mathrm{~mJ}$ levels, however, produced pronounced darkening but less reddening. Since the TEM images show that irradiations at both levels have produced iron grains with similar size distributions $(<30 \mathrm{~nm})$, the difference must have been caused by thicker iron-bearing glassy layers produced at higher energy levels. Although the npFe ${ }^{0}$ abundances produced by $50 \mathrm{~mJ}$ levels are higher than that produced by $25 \mathrm{~mJ}$ levels, the reddening effects caused by npFe ${ }^{0}$ must have been suppressed by thicker amorphous layers which have profound darkening effects on reflectance.

In contrast to the visible and near-infrared spectra, MIR spectral features are much more immune to the intense irradiations. Although the Christiansen Feature and many reflectance bands show a redshift of up to $6 \mathrm{~cm}^{-1}$ under the most intense irradiations, the overall spectral features can survive the strongest irradiations which may be much more intense than the realistic micrometeorite bombardment levels on asteroid surface (e.g., Clark et al. 2002; Markley \& Kletetschka 2016). The much lower MIR reflectance of samples irradiated at $50 \mathrm{~mJ}$ as compared with that irradiated at $25 \mathrm{~mJ}$ is again attributed to the thicker amorphous layers produced at higher energy irradiations.

Reflectance modeling results indicate that the MIR features are not susceptible to the productions of the $n \mathrm{pFe}^{0}$ and as long as the glassy coating thickness is less than $200 \mathrm{~nm}$ the CF position and other spectral features can be retained. The fact that the olivine crystal structure can survive heavy irradiations is supported by XRD and Raman scattering measurements. No detectable crystal structural changes were found in the XRD spectra of heavily irradiated samples. Although Raman peaks of the heavily irradiated samples show broadening and redshifts compared with the original sample, indicating the productions of glassy materials, lattice defects, and tensile stress, all vibrational modes can be clearly recognized.

As pyroxene is known to be more resistant to space weathering modifications (Yamada et al. 1999), the current results show that MIR spectral features may be very useful in remotely identifying the surface mineralogical compositions of airless bodies undergoing strong space weathering modifications.

Acknowledgements. We thank Sho Sasaki for helpful suggestions in the pulsed laser simulation system setup and Weidong Jin for programming the translation stage. Constructive reviews by Tomáš Kohout improved the quality of the manuscript. This work was supported by the National Natural Science Foundation of China (U1631124, 11573058, 11327303) and by the Fundamental Research Funds for the Central Universities, China University of Geosciences (Wuhan)

\section{References}

Beichman, C., Bryden, G., Gautier, T., et al. 2005, ApJ, 626, 1061

Berger, E. L., \& Keller, L. P. 2015, in LPSC, 46th Lunar and Planetary Science Conference, 2351

Bohren, C. F., \& Huffman, D. R. 2008, in Absorption and scattering of light by small particles (John Wiley \& Sons)

Britt, D., Kohout, T., Schelling, P., \& Consolmagno, G. J. 2014, in AAS/DPS Meeting, 46

Brucato, J., Strazzulla, G., Baratta, G., \& Colangeli, L. 2004, A\&A, 413, 395

Brunetto, R., Romano, F., Blanco, A., et al. 2006, Icarus, 180, 546

Burns, R. G. 1993, Mineralogical applications of crystal field theory (Cambridge University Press)

Cerdeira, F., Buchenauer, C., Pollak, F. H., \& Cardona, M. 1972, Phys. Rev. B, 5,580
Chapman, C. R. 1996, Meteorit. Planet. Sci., 31, 699

Chapman, C. R. 2004, Annu. Rev. Earth Planet. Sci., 32, 539

Clark, R. N., \& Roush, T. L. 1984, J. Geophys. Res.: Solid Earth, 89, 6329

Clark, B. E., Hapke, B., Pieters, C., \& Britt, D. 2002, Asteroids III, eds. W. F Bottke Jr., A. Cellino, P. Paolischi, \& R. P. Binzel (Tucson: University of Arizona Press), 585

Denevi, B., Lucey, P., \& Sherman, S. 2008, J. Geophys. Res.: Planets, 113, E02003

Dorschner, J., Begemann, B., Henning, T., Jaeger, C., \& Mutschke, H. 1995, A\&A, 300, 503

Fabian, D., Jäger, C., Henning, T., Dorschner, J., \& Mutschke, H. 2000, A\&A, 364,282

Fabian, D., Henning, T., Jäger, C., et al. 2001, A\&A, 378, 228

Fulvio, D., Brunetto, R., Vernazza, P., \& Strazzulla, G. 2012, A\&A, 537, L11

Gaffey, M. J. 2010, Icarus, 209, 564

Hamilton, V. E. 2010, Chem. Erde-Geochem., 70, 7

Hapke, B. 1973, The Moon, 7, 342

Hapke, B. 1981, J. Geophys. Res.: Solid Earth, 86, 3039

Hapke, B. 2001, J. Geophys. Res.: Planets, 106, 10039

Hapke, B. 2012, Theory of reflectance and emittance spectroscopy (Cambridge University Press)

Hapke, B. 2013, J. Quant. Spectr. Rad. Transf., 116, 184

Hapke, B., Cassidy, W., \& Wells, E. 1975, The Moon, 13, 339

Hofmeister, A. M. 1987, Phys. Chem. Miner., 14, 499

Jäger, C., Mutschke, H., Begemann, B., Dorschner, J., \& Henning, T. 1994, A\&A, 292, 641

Keller, L. P., \& McKay, D. S. 1993, Science, 261, 1305

Keller, L. P., \& McKay, D. S. 1997, Geochim. Cosmochim. Acta, 61, 2331

Kohout, T., Čuda, J., Filip, J., et al. 2014, Icarus, 237, 75

Kolesov, B., \& Geiger, C. 2004, Phys. Chem. Miner., 31, 142

Kuhlman, K. R., Sridharan, K., \& Kvit, A. 2015, Planet. Space Sci., 115, 110

Lawrence, S. J., \& Lucey, P. G. 2007, J. Geophys. Res.: Planets, 112, E07005

Loeffler, M., Baragiola, R., \& Murayama, M. 2008, Icarus, 196, 285

Lucey, P. G., \& Riner, M. A. 2011, Icarus, 212, 451

Lucey, P. G., Greenhagen, B. T., Song, E., et al. 2016, Icarus, 283, 343

Marchi, S., Brunetto, R., Magrin, S., Lazzarin, M., \& Gandolfi, D. 2005, A\&A, 443, 769

Markley, M., \& Kletetschka, G. 2016, Icarus, 268, 204

Miller, K. A., De Ruette, N., Harlow, G., Domingue, D. L., \& Savin, D. W. 2014, in AAS Meet., 224

Mishchenko, M. I., Dlugach, J. M., Yanovitsku, E. G., \& Zakharova, N. T. 1999,

Morris, R. 1980, in LPSC, 11, 1697

Mustard, J. F., \& Hays, J. E. 1997, Icarus, 125, 145

Nesvornỳ, D., Jedicke, R., Whiteley, R. J., \& Ivezić, Ž. 2005, Icarus, 173, 132

Noel, Y., Catti, M., D’Arco, P., \& Dovesi, R. 2006, Phys. Chem. Miner., 33, 383

Noguchi, T., Nakamura, T., Kimura, M., et al. 2011, Science, 333, 1121

Ordal, M. A., Bell, R. J., Alexander, R. W., Newquist, L. A., \& Querry, M. R. 1988, Appl. Opt., 27, 1203

Paques-Ledent, M. T., \& Tarte, P. 1973, Spectrochim. Acta A, 29, 1007

Pieters, C. M. 1998, Int. Geol. Rev., 40, 981

Pieters, C. M. 2002, in LPI Mars Spectral Workshop II, 33rd Annual Lunar and Planetary Science Conf., March 11-15, Hauston, Texas, 177

Pieters, C. M., Taylor, L. A., Noble, S. K., et al. 2000, Meteorit. Planet. Sci., 35, 1101

Poulet, H., \& Mathieu, J. P. 1976, Vibration spectra and symmetry of crystals (Gordon \& Breach Science Pub.)

Quirantes, A., \& Bernard, S. 2004, J. Quant. Spectr. Rad. Transf., 89, 311

Salisbury, J. W., Basu, A., \& Fischer, E. M. 1997, Icarus, 130, 125

Sasaki, S., Nakamura, K., Hamabe, Y., Kurahashi, E., \& Hiroi, T. 2001, Nature, 410,555

Sasaki, S., Hiroi, T., Nakamura, K., et al. 2002, Adv. Space Res., 29, 783

Sasaki, S., Kurahashi, E., Yamanaka, C., \& Nakamura, K. 2003, Adv. Space Res., 31,2537

Shkuratov, Y., Kaydash, V., Korokhin, V., et al. 2012, J. Quant. Spectr. Rad. Transf., 113, 2431

Stamnes, K., Tsay, S.-C., Wiscombe, W., \& Laszlo, I. 2000, DISORT, Goddard Space flight center, NASA

Tang, H., Wang, S., \& Li, X. 2012, Planet. Space Sci., 60, 322

Taylor, L. A., Pieters, C. M., Keller, L. P., Morris, R. V., \& McKay, D. S. 2001, J. Geophys. Res.: Planets, 106, 27985

Vernazza, P., Carry, B., Emery, J., et al. 2010, Icarus, 207, 800

Yamada, M., Sasaki, S., Nagahara, H., et al. 1999, Earth Planets Space, 51, 1255

Zhang, H., \& Voss, K. J. 2011, Icarus, 215, 27

Zhang, H., Yang, Y., Jin, W., Liu, C., \& Hsu, W. 2014, Opt. Express, 22, 21280 